\title{
Multiple scales and singular limits of perfect fluids
}

\author{
NILASIS CHAUDHURIID
}

Abstract. In this article, our goal is to study the singular limits for a scaled barotropic Euler system modeling a rotating, compressible and inviscid fluid, where Mach number $=\epsilon^{m}$, Rossby number $=\epsilon$ and Froude number $=\epsilon^{n}$ are proportional to a small parameter $\epsilon \rightarrow 0$. The fluid is confined to an infinite slab, the limit behavior is identified as the incompressible Euler system or a damped incompressible Euler system depending on the relation between $m$ and $n$. For well-prepared initial data, the convergence is shown on the lifespan time interval of the strong solutions of the target system, whereas a class of generalized dissipative solutions is considered for the primitive system. The technique can be adapted to the compressible NavierStokes system in the subcritical range of the adiabatic exponent $\gamma$ with $1<\gamma \leq \frac{3}{2}$, where weak solutions are not known to exist.

\section{Introduction}

We study the model of a rotating fluid as described in Chemin et al. [13]. Let $T>0$ and $\Omega=\mathbb{R}^{2} \times(0,1) \subset \mathbb{R}^{3}$ be an infinite slab. We consider the scaled compressible Euler equation in the time-space cylinder $Q_{T}=(0, T) \times \Omega$ describing the time evolution of the mass density $\varrho=\varrho(t, x)$ and the momentum field $\mathbf{m}=\mathbf{m}(t, x)$ of a rotating inviscid fluid with axis of rotation $\mathbf{b}=(0,0,1)$ :

- Conservation of mass

$$
\partial_{t} \varrho+\operatorname{div}_{x} \mathbf{m}=0 .
$$

- Conservation of momentum

$$
\partial_{t} \mathbf{m}+\operatorname{div}_{x}\left(\frac{\mathbf{m} \otimes \mathbf{m}}{\varrho}\right)+\frac{1}{\mathrm{Ma}^{2}} \nabla_{x} p(\varrho)+\frac{1}{\mathrm{Ro}} \mathbf{b} \times \mathbf{m}=\frac{1}{\mathrm{Fr}^{2}} \varrho \nabla_{x} G,
$$

where $p$ stands for pressure and $G$ is the gravitational potential that we consider independent of time.

- Pressure law The pressure $p$ and the density $\varrho$ of the fluid are interrelated by the standard isentropic law

$$
p(\varrho)=a \varrho^{\gamma}, a>0, \gamma>1 .
$$

Mathematics Subject Classification: Primary: 76U05; Secondary: 35Q35, 35D99, 76N10

Keywords: Compressible Euler system, Rotating fluids, Dissipative solution, Low Mach and Rossby number limit, Multiple scales, Compressible Navier-Stokes system. 
- Boundary condition Here we consider an impermeability condition on the horizontal boundary, i.e.,

$$
\mathbf{m} \cdot \mathbf{n}=0, \mathbf{n}=(0,0, \pm 1) .
$$

- The scaled system contains characteristic numbers:

Ma- Mach number,

Ro- Rossby number,

Fr- Froude number.

Here we consider the following scaling:

$$
\mathrm{Ma} \approx \epsilon^{m}, \text { Ro } \approx \epsilon, \text { Fr } \approx \epsilon^{n} \text { for } \epsilon>0, m, n>0
$$

- Far field condition The condition is needed as we consider the unbounded domain $\Omega$. Let us introduce the notation $x=\left(x_{h}, x_{3}\right)$ and $P_{h}(x)=x_{h}$. For each $\epsilon>0$, we identify a static solution that satisfies (1.1)-(1.2) with (1.5). More precisely, a static solution is a pair $\left(\tilde{\varrho}_{\epsilon}, \mathbf{0}\right)$, where the density profile $\tilde{\varrho}_{\epsilon}$ satisfies

$$
\nabla_{x} p\left(\tilde{\varrho}_{\epsilon}\right)=\epsilon^{2(m-n)} \tilde{\varrho}_{\epsilon} \nabla_{x} G .
$$

In general, there are infinitely many static solutions for a given potential $G$. We introduce a far field condition as

$$
\left|\varrho_{\epsilon}-\tilde{\varrho}_{\epsilon}\right| \rightarrow 0, \mathbf{m}_{\epsilon} \rightarrow \mathbf{0} \text { as }\left|x_{h}\right| \rightarrow \infty
$$

where $\left(\varrho_{\epsilon}, \mathbf{m}_{\epsilon}\right)$ indicates that state variables depend on the parameter $\epsilon$.

- Initial data For each $\epsilon>0$, we supplement the initial data as

$$
\varrho_{\epsilon}(0, \cdot)=\varrho_{\epsilon, 0}, \mathbf{m}_{\epsilon}(0, \cdot)=\mathbf{m}_{\epsilon, 0} .
$$

- Choice of $G$ As a matter of fact, the gravitational potential $G$ can be seen as a sum of the centrifugal force proportional to the norm of the horizontal component of the spatial variable, i.e., $\left(x_{1}^{2}+x_{2}^{2}\right)$ and the gravitational force acting in the vertical direction $x_{3}$. We omit the effect of the centrifugal force in the present paper motivated by certain meteorological models. Instead we consider

$$
G(x)=-x_{3} \text { in } \Omega
$$

corresponding to the gravitational force acting in the vertical direction.

Multiple scaling We consider singular limit problems for $\epsilon \rightarrow 0$ in two multi-scale regimes:

- Case I Here $m, n$ are interrelated by

$$
\frac{m}{2}>n \geq 1
$$

It implies that we are interested in low Mach and Rossby number limit in the presence of low stratification. 
- Case II In this case, we consider $m=n=1$, that implies the low Mach and Rossby number limit in the presence of strong stratification.

Thus, we study the effect of the low Mach number limit (also called incompressible limit), the low Rossby number limit and the low Froude number limit acting simultaneously on the system (1.1)-(1.2).

Formally, we observe that the low Mach number limit regime indicates that the fluid becomes incompressible and the low Rossby number limit indicates the fast rotation of the fluid and as a consequence of that the fluid becomes planner (two-dimensional).

Since solutions of the (primitive) compressible Euler systems are expected to develop singularities (shock waves) in a finite time, there are two approaches to deal with the singular limit problem.

I. The first approach is to consider classical (strong) solutions of the primitive system and expect them to converge to the classical solutions of the target system. Here the most important and highly non-trivial problem is to ensure that the lifespan of the strong solutions with respect to the singular parameter $(\epsilon)$ are uniformly bounded below and away from zero.

II. The second approach is based on the concept of weak, measure-valued or dissipative solutions of the primitive system. Given a suitable choice of initial data, one can show convergence provided that the target system admits a smooth solution.

For the first approach in the low Mach number limits, we have results of Ebin [14], Kleinermann and Majda [28], Schochet [34], and many others. For rotating fluids, there are results by Babin et al. [3,4] and Chemin et al. [13].

In the case of the second approach, most results dealing with weak solutions have been studied for the compressible Navier-Stokes system with additional consideration of the high Reynolds number limit. For rotating fluids, there are several results, see Feireisl, Gallagher and Novotný [18], Feireisl et al. [17], Feireisl and Novotný [24,25] and $\mathrm{Li}[29]$.

The existence of a global-in-time weak solution of the compressible Euler equation satisfying the energy inequality is still open for general initial data. Therefore, it is important to consider a measure-valued solution or a newly developed dissipative solution for this system. The concept of measure-valued solutions has been studied in various contexts, such as in the analysis of numerical schemes, etc. In the following articles by Alibert and Bouchitté [2], Gwiazda et al. [26], Březina and Feireisl [8], Březina [10], Basarić [5] et al. [21], we observe the development of the theory of measure-valued solution for various models describing compressible fluids, mainly using Young measures.

Recently Feireisl et al. [22] and Breit et al. [6] have given a new definition of generalized solutions for the compressible Euler system, which they call a dissipative solution. This new definition does not include Young measures.

The advantages of the second approach to singular limit problems are as follows: 
- Weak or measure-valued solutions to the primitive system exist globally in time. Thus, the result depends only on the lifespan of the target problem, which may be finite.

- Since convergence holds for a large class of generalized solutions, this suggests some stability of the limit solution of the target system.

In particular, the results with generalized solutions are better in the sense that the convergence holds for a larger class of solutions and for the lifespan of the limit system.

There are a number of articles dealing with the low Mach number limit in the context of measure-valued solutions. In Feireisl et al. [20], Bruell and Feireisl [9], Březina and Mácha [11], it is shown that measure-valued solution of the primitive system, describing a compressible inviscid fluid, converges to a strong solution of the incompressible target system given suitable initial data. The 'single-scale' limit of our system, i.e., $m=1$ and $G=0$, was studied by Nečasová and Tong [31], again using the measure-valued solutions.

The framework of measure-valued solutions can also be applied in the context of the Navier-Stokes system. Although global in time weak solutions exist here, their existence is constrained by the technical condition for the adiabatic exponent $\gamma>\frac{3}{2}$. To deal with this technical constraint, Feireisl et al. [19] introduced the concept of dissipative measure-valued solution in terms of the Young measure. Here we use a slightly different approach, introducing a dissipative solution for Navier-Stokes system without explicit presence of the Young measure. In this way, we extend the convergence result to the Navier-Stokes system with high Reynolds number limit in the regime where the existence of weak solutions is unknown.

In our approach, it is very important to consider correct initial data, which are mainly called well-prepared and ill-prepared initial data. Feireisl and Novotný explain in [23] that for ill-prepared data the presence of Rossby acoustic waves plays an important role in the analysis of singular limits. For well-prepared data, on the other hand, this effect is absent. Here we are concerned with the well-prepared initial data.

Our main goal is to prove that under suitable choice of initial data a dissipative solution of a compressible rotating Euler system in low Mach and low Rossby regime converges to a strong solution of the incompressible Euler system in 2D. Hence, our plan for the article is following:

1. Derivation of limit systems.

2. Solutions of the target and primitive systems and relative energy inequality.

3. Singular limit for 'well-prepared' data.

4. Extension to the Navier-Stokes system.

\subsection{Notation and preliminaries}

- Here we consider the pressure potential $(P)$ as

$$
P(\varrho)=\frac{a}{\gamma-1} \varrho^{\gamma}
$$


for the pressure law (1.3). Without loss of generality, we assume the 'normalized' setting for $p$ as

$$
p^{\prime}(1)=1 \text {. }
$$

Consideration of (1.9) leads to the following relations:

$$
\varrho P^{\prime}(\varrho)-P(\varrho)=p(\varrho) \text { and } \varrho P^{\prime \prime}(\varrho)=p^{\prime}(\varrho) \text { for } \varrho>0 \text {. }
$$

- Let $r$ be a positive real-valued function and its range lie in a compact subset of $(0, \infty)$. Then for any $\varrho \geq 0$, there exists $r_{1}, r_{2}>0$, that depend on $r$ such that

$$
P(\varrho)-(\varrho-r) P^{\prime}(r)-P(r) \geq c(r)\left\{\begin{array}{l}
(\varrho-r)^{2} \text { for } r_{1} \leq \varrho<r_{2} \\
1+\varrho^{\gamma}, \text { otherwise }
\end{array}\right.
$$

where $c(r)$ is a constant dependent on $r$. We note that if $\gamma>2$, one can consider $1+\varrho^{2}$ instead of $1+\varrho^{\gamma}$ in (1.10). Taking the last observation into account, we can replace $\gamma$ by $\gamma^{\prime}=\min \{2, \gamma\}$ in (1.10).

- Essential and residual part of a function: We introduce a function $\chi=\chi(\varrho)$ such that

$$
\chi(\cdot) \in C_{c}^{\infty}(0, \infty), 0 \leq \chi \leq 1, \chi(\varrho)=1 \text { if } \varrho_{1} \leq \varrho \leq \varrho_{2},
$$

where $\varrho_{1}, \varrho_{2}>0$. For a function $H=H(\varrho, \mathbf{u})$, we set

$$
[H]_{\mathrm{ess}}=\chi(\varrho) H(\varrho, \mathbf{u}),[H]_{\mathrm{res}}=(1-\chi(\varrho)) H(\varrho, \mathbf{u}), \varrho \geq 0 \text { and } \mathbf{u} \in \mathbb{R}^{3} \text {. }
$$

\section{Derivation of the target system}

In this section, we provide an informal justification how we obtain the target system. First we note that $\left(\tilde{\varrho}_{\epsilon}, \mathbf{0}\right)$ is a static state solution for (1.1)-(1.2) and it satisfies

$$
\nabla_{x} p\left(\tilde{\varrho}_{\epsilon}\right)=\epsilon^{2(m-n)} \tilde{\varrho}_{\epsilon} \nabla_{x} G
$$

Let us consider the following asymptotic expansion:

$$
\begin{aligned}
& \varrho_{\epsilon}=\tilde{\varrho}_{\epsilon}+\epsilon^{m} \varrho_{\epsilon}^{(1)}+\epsilon^{2 m} \varrho_{\epsilon}^{(2)}+\cdots, \\
& \mathbf{m}_{\epsilon}=\tilde{\varrho}_{\epsilon} \mathbf{v}+\epsilon^{m} \mathbf{m}_{\epsilon}^{(1)}+\epsilon^{2 m} \mathbf{m}_{\epsilon}^{(2)}+\cdots .
\end{aligned}
$$

As a consequence of the above and Taylor's expansion, we obtain

$$
p\left(\varrho_{\epsilon}\right)=p\left(\tilde{\varrho}_{\epsilon}\right)+\epsilon^{m} p^{\prime}\left(\tilde{\varrho}_{\epsilon}\right) \varrho_{\epsilon}^{(1)}+\epsilon^{2 m}\left(p^{\prime}\left(\tilde{\varrho}_{\epsilon}\right) \varrho_{\epsilon}^{(2)}+\frac{1}{2} p^{\prime \prime}\left(\tilde{\varrho}_{\epsilon}\right)\left(\varrho_{\epsilon}^{(1)}\right)^{2}\right)+o\left(\epsilon^{3 m}\right) .
$$


2.1. Case I: $\frac{m}{2}>n>1$; target system: Euler system

Clearly conditions on $m$ and $n$ in (1.8) indicate $\lim _{\epsilon \rightarrow 0} \nabla_{x} P^{\prime}\left(\tilde{\varrho}_{\epsilon}\right)=0$. Without loss of generality, we assume

$$
\tilde{\varrho}_{\epsilon} \approx \bar{\varrho}+\epsilon^{2(m-n)}, \text { where } \bar{\varrho} \text { is a positive constant. }
$$

Thus, we rewrite the continuity equation and the momentum equation as

$$
\bar{\varrho} \operatorname{div}_{x} \mathbf{v}+\epsilon^{m}\left(\partial_{t} \varrho_{\epsilon}^{(1)}+\operatorname{div}_{x}\left(\mathbf{m}_{\epsilon}^{(1)}\right)\right)+o\left(\epsilon^{2 m}\right)=0
$$

and

$$
\begin{gathered}
\bar{\varrho}\left(\partial_{t} \mathbf{v}+\left(\mathbf{v} \cdot \nabla_{x}\right) \mathbf{v}\right)+\nabla_{x}\left(p^{\prime}(\bar{\varrho}) \varrho_{\epsilon}^{(2)}+\frac{1}{2} p^{\prime \prime}(\bar{\varrho})\left(\varrho_{\epsilon}^{(1)}\right)^{2}\right)+\epsilon^{m-1} \mathbf{b} \times \mathbf{m}_{\epsilon}^{(1)} \\
+\frac{1}{\epsilon^{m}} p^{\prime}(\bar{\varrho}) \nabla_{x} \varrho_{\epsilon}^{(1)}+\frac{1}{\epsilon}(\varrho \bar{b} \times \mathbf{v})-\epsilon^{m-2 n} \varrho_{\epsilon}^{(1)} \nabla_{x} G+o(\epsilon)=0,
\end{gathered}
$$

respectively. Let $\mathbb{H}$ be the Helmholtz projection, then we have

$$
\mathbb{H}\left(\partial_{t}\left(\varrho_{\epsilon} \mathbf{u}_{\epsilon}\right)+\operatorname{div}_{x}\left(\frac{\mathbf{m}_{\epsilon} \otimes \mathbf{m}_{\epsilon}}{\varrho_{\epsilon}}\right)+\frac{1}{\epsilon} \mathbf{b} \times \varrho_{\epsilon} \mathbf{u}_{\epsilon}\right)=\mathbb{H}\left(\frac{1}{\epsilon^{2 n}} \varrho_{\epsilon} \nabla_{x} G\right) .
$$

Suppose $\mathbf{m}_{\epsilon} \rightarrow \bar{\varrho} \mathbf{v}$ in a strong sense, multiplying the above equation by $\epsilon$ and using our standard expansion technique, we obtain

$$
\mathbb{H}[\mathbf{b} \times \bar{\varrho} \mathbf{v}]=0 .
$$

From the above equations, we get the following relations:

$\mathbf{b} \times \mathbf{v}=\nabla_{x} \psi$, for a scalar valued function $\psi$ with

$$
\begin{aligned}
& \psi_{x_{3}}=0, \psi(x)=\psi\left(x_{h}\right), \nabla_{x_{h}}^{\perp} \psi=\mathbf{v}_{h}, \mathbf{v}_{h}=\left(v_{1}, v_{2}\right), \\
& \operatorname{div}_{x_{h}} \mathbf{v}_{h}=0, v_{x_{x_{3}}}=0, \text { with } \nabla_{x_{h}}^{\perp} \equiv\left(-\frac{\partial}{\partial x_{2}}, \frac{\partial}{\partial x_{1}}\right) .
\end{aligned}
$$

Thus, we have

$$
v_{1_{x_{3}}}=0, v_{x_{3}}=0 \text {, and } \mathbf{v}(x)=\mathbf{v}\left(x_{h}\right) .
$$

The boundary condition (1.4) leads us the conclusion $v_{3}\left(x_{h}, x_{3}\right)=0$ and $\mathbf{v}=$ $\left(\mathbf{v}_{h}\left(x_{h}\right), 0\right)$. Finally, the above discussion yields the target system

$$
\begin{aligned}
& \operatorname{div}_{x_{h}} \mathbf{v}_{h}=0, \\
& \partial_{t} \mathbf{v}_{h}+\left(\mathbf{v}_{h} \cdot \nabla_{x_{h}}\right) \mathbf{v}_{h}+\nabla_{x_{h}} \Pi=0,
\end{aligned}
$$

i.e., the incompressible Euler equation in 2D. 
2.2. Case II: $m=n=1$; target system: damped Euler system describing a stratified fluid

In this case, we first recall that, a static solution satisfies

$$
\nabla_{x} p\left(\tilde{\varrho}_{\epsilon}\right)=\tilde{\varrho}_{\epsilon} \nabla_{x} G
$$

It follows that $\tilde{\varrho}_{\epsilon}$ is independent of $\epsilon$ and we denote it by $\hat{\varrho}$. Therefore, we rewrite the continuity equation and the momentum equation as

$$
\operatorname{div}_{x}(\hat{\varrho} \mathbf{v})+\epsilon\left(\partial_{t} \varrho_{\epsilon}^{(1)}+\operatorname{div}_{x}\left(\mathbf{v}_{\epsilon}^{(1)} \hat{\varrho}+\mathbf{v} \varrho_{\epsilon}^{(1)}\right)\right)+o\left(\epsilon^{2}\right)=0
$$

and

$$
\begin{gathered}
\partial_{t}(\hat{\varrho} \mathbf{v})+\operatorname{div}_{x}(\hat{\varrho} \mathbf{v} \otimes \mathbf{v})+\nabla_{x}\left(p^{\prime}(\hat{\varrho}) \varrho_{\epsilon}^{(2)}+\frac{1}{2} p^{\prime \prime}(\hat{\varrho})\left(\varrho_{\epsilon}^{(1)}\right)^{2}\right)+\mathbf{b} \times \mathbf{m}_{\epsilon}^{(1)} \\
+\frac{1}{\epsilon}\left(\nabla_{x}\left(p^{\prime}(\hat{\varrho}) \varrho_{\epsilon}^{(1)}\right)+\mathbf{b} \times \hat{\varrho} \mathbf{v}-\varrho_{\epsilon}^{(1)} \nabla_{x} G\right)+o(\epsilon)=0
\end{gathered}
$$

respectively, where $\mathbf{m}_{\epsilon}^{(1)}=\mathbf{v}_{\epsilon}^{(1)} \hat{\varrho}+\mathbf{v} \varrho_{\epsilon}^{(1)}$. have

We assume $\left(\frac{\varrho_{\epsilon}-\hat{\varrho}}{\epsilon}\right) \rightarrow q$ and $\mathbf{u}_{\epsilon} \rightarrow \mathbf{v}$ in a strong sense. Then as a consequence we

$$
\begin{aligned}
& \nabla_{x}\left(p^{\prime}(\hat{\varrho}) q\right)+\mathbf{b} \times \hat{\varrho} \mathbf{v}=q \nabla_{x} G \\
& \operatorname{div}_{x}(\hat{\varrho} \mathbf{v})=0 \\
& \partial_{t}(\hat{\varrho} \mathbf{v})+\operatorname{div}_{x}(\hat{\varrho} \mathbf{v} \otimes \mathbf{v})+\nabla_{x} \Pi_{1}+\mathbf{b} \times \mathbf{m}_{1}=0 \\
& \partial_{t} q+\operatorname{div}_{x} \mathbf{m}_{1}=0 .
\end{aligned}
$$

Here we get $\left(P^{\prime \prime}(\hat{\varrho}) q\right)_{x_{3}}=0$ and this implies $\left(P^{\prime \prime}(\hat{\varrho}) q\right)\left(x_{h}, x_{3}\right)=\left(P^{\prime \prime}(\hat{\varrho}) q\right)\left(x_{h}, 0\right)$ for $x_{3} \in(0,1)$. Consequently, from the choice of $G$, we obtain

$$
q\left(x_{h}, x_{3}\right)=f\left(\hat{\varrho}\left(x_{3}\right)\right) \bar{q}\left(x_{h}\right)
$$

where $f$ is a bounded continuous function in $(0,1)$. Without loss of generality, we assume $\hat{\varrho}>0$. Finally, we summarize the above discussion and derive the expected target system as

$$
\begin{aligned}
& \nabla_{x_{h}}\left(P^{\prime \prime}(\hat{\varrho}) q\right)+\mathbf{b} \times \mathbf{v}=0 \\
& \partial_{t}\left(\Delta_{x_{h}}\left(p^{\prime}(\hat{\varrho}) q\right)-q\right)+\nabla_{x_{h}}^{\perp}\left(p^{\prime}(\hat{\varrho}) q\right) \cdot \nabla_{x_{h}}\left(\Delta_{x_{h}}\left(P^{\prime \prime}(\hat{\varrho}) q\right)\right)=0,
\end{aligned}
$$

in $\mathbb{R}^{2}$, for each $x_{3} \in(0,1)$ and $q$ in the form given in (2.3). The system (2.4) represents a stratified fluid in $\mathbb{R}^{2} \times(0,1)$ where the time evolution is given in $\mathbb{R}^{2}$ only and $q$ can be viewed as a stream function of the flow. 


\section{Solvability of target systems, primitive system and relative energy}

\subsection{Target system: existence of the strong solution}

In the last section, we informally obtained the expected target systems in both cases. Here we state some well-known results that prove the existence of a strong solution for these systems.

\subsubsection{Case I}

First, we recall the expected target system, the 2D Euler equation, i.e.,

$$
\begin{aligned}
& \operatorname{div}_{x_{h}} \mathbf{v}_{h}=0, \text { in } \mathbb{R}^{2}, \\
& \partial_{t} \mathbf{v}_{h}+\left(\mathbf{v}_{h} \cdot \nabla_{x_{h}}\right) \mathbf{v}_{h}+\nabla_{x_{h}} \Pi=0, \text { in } \mathbb{R}^{2} .
\end{aligned}
$$

The following result of Kato and Lai [27] ensures existence and uniqueness for the incompressible Euler system in $\mathbb{R}^{2}$ for sufficiently smooth initial data.

Proposition 3.1. Let

$$
\mathbf{v}_{0} \in W^{k, 2}\left(\mathbb{R}^{2} ; \mathbb{R}^{2}\right), k \geq 3, \operatorname{div}_{x_{h}} \mathbf{v}_{0}=0
$$

be given. Then the system (2.2) supplemented with the initial data $\mathbf{v}_{h}(0)=\mathbf{v}_{0}$ admits a regular solution $\left(\mathbf{v}_{h}, \Pi\right)$, unique in the class

$$
\begin{aligned}
\mathbf{v}_{h} & \in C\left([0, T] ; W^{k, 2}\left(\mathbb{R}^{2} ; \mathbb{R}^{2}\right)\right), \partial_{t} \mathbf{v}_{h} \in C\left([0, T] ; W^{k-1,2}\left(\mathbb{R}^{2} ; \mathbb{R}^{2}\right)\right), \\
\Pi & \in C\left([0, T] ; W^{k, 2}\left(\mathbb{R}^{2}\right)\right),
\end{aligned}
$$

with div $x_{h} \mathbf{v}_{h}=0$.

Alternatively, we write the system (2.2) as

$$
\partial_{t} \operatorname{curl}_{x_{h}} \mathbf{v}_{h}+\mathbf{v}_{h} \cdot \nabla_{x_{h}} \operatorname{curl}_{x_{h}} \mathbf{v}_{h}=0, \text { in } \mathbb{R}^{2},
$$

i.e., vorticity formulation of the system (2.2) where the vorticity is $\operatorname{curl}_{x_{h}} \mathbf{v}_{h}$.

\subsubsection{Case II}

Now we consider the case $m=n=1$. Here a static solution $\tilde{\varrho}_{\epsilon}$ is independent of $\epsilon$ and in particular, we choose a static solution $\left(\hat{\varrho}=\hat{\varrho}\left(x_{3}\right)\right)$ such that

$$
0<\hat{\varrho} \in C^{3}(\Omega) \cap W^{1, \infty}(\Omega) .
$$

In this case, that expected target system is a damped variant of incompressible Euler system. It states that for each $x_{3} \in[0,1],\left(q\left(x_{h}, x_{3}\right), \mathbf{v}\left(x_{h}, x_{3}\right)\left(=\mathbf{v}_{h}\left(x_{h}\right), 0\right)\right)$ solves

$$
\nabla_{x}\left(P^{\prime \prime}\left(\hat{\varrho}\left(x_{3}\right)\right) q\right)+\mathbf{b} \times \mathbf{v}=0
$$




$$
\partial_{t}\left(\Delta_{x_{h}}\left(p^{\prime}\left(\hat{\varrho}\left(x_{3}\right)\right) q\right)-q\right)+\nabla_{x_{h}}^{\perp}\left(p^{\prime}\left(\hat{\varrho}\left(x_{3}\right)\right) q\right) \cdot \nabla_{x_{h}}\left(\Delta_{x_{h}}\left(P^{\prime \prime}\left(\hat{\varrho}\left(x_{3}\right)\right) q\right)\right)=0,
$$

supplemented with initial data $q(0, \cdot)=q_{0}$ in $\Omega$.

If assume $q_{0} \in W^{k, 2}\left(\mathbb{R}^{2}\right)$ with $k>4$, then for each $x_{3} \in[0,1]$, Eq. (3.3) admits strong solution. Furthermore, following Oliver [33, Theorem 3], we have for each $x_{3} \in[0,1], q\left(\cdot, x_{3}\right) \in C\left([0, T] ; W^{k, 2}\left(\mathbb{R}^{2}\right)\right) \cap C^{1}\left([0, T] ; W^{k-1,2}\left(\mathbb{R}^{2}\right)\right)$.

Finally, from (2.3) we state the regularity of the target system as follows:

Proposition 3.2. Suppose that

$$
q_{0} \in W^{k, 2}\left(\mathbb{R}^{2}\right) \text { for } k \geq 4 .
$$

Then, the problem (2.4) admits a solution q, unique in the class

$$
q \in C\left([0, T] ; W^{k, 2}\left(\mathbb{R}^{2}\right) \times C^{3}([0,1])\right) \cap C^{1}\left([0, T] ; W^{k-1,2}\left(\mathbb{R}^{2}\right) \times C^{3}([0,1])\right) .
$$

3.2. Definition of a dissipative solution for the primitive system

Now we provide the definition of a dissipative solution for the primitive system of our consideration.

Definition 3.3. Let $\epsilon>0$ and $\tilde{\varrho}_{\epsilon}>0$. We say $\left(\varrho_{\epsilon}, \mathbf{u}_{\epsilon}\right)$ with

$$
\begin{aligned}
& \varrho_{\epsilon}-\tilde{\varrho}_{\epsilon} \in C_{\text {weak }}\left([0, T] ; L^{2}+L^{\gamma}(\Omega)\right), \\
& \varrho_{\epsilon} \geq 0, \mathbf{m}_{\epsilon} \in C_{\text {weak }}\left([0, T] ; L^{2}+L^{\frac{2 \gamma}{\gamma+1}}(\Omega)\right),
\end{aligned}
$$

is a dissipative solution to the compressible Euler equation (1.1)-(1.7) with initial data $\left(\varrho_{0, \epsilon},(\varrho \mathbf{u})_{0, \epsilon}\right)$ satisfying

$$
\begin{aligned}
\varrho_{0, \epsilon} \geq 0, E_{0, \epsilon} & =\int_{\Omega}\left(\frac{1}{2} \frac{\left|\mathbf{m}_{0, \epsilon}\right|^{2}}{\varrho_{0, \epsilon}}+P\left(\varrho_{0, \epsilon}\right)-\left(\varrho_{0, \epsilon}-\tilde{\varrho}_{\epsilon}\right) P^{\prime}\left(\tilde{\varrho}_{\epsilon}\right)-P\left(\tilde{\varrho}_{\epsilon}\right)\right) \mathrm{d} x \\
& <\infty,
\end{aligned}
$$

if there exist the turbulent defect measures

$$
\mathfrak{R}_{m_{\epsilon}} \in L^{\infty}\left(0, T ; \mathcal{M}^{+}\left(\bar{\Omega} ; \mathbb{R}_{\mathrm{sym}}^{d \times d}\right)\right), \mathfrak{R}_{e_{\epsilon}} \in L^{\infty}\left(0, T ; \mathcal{M}^{+}(\bar{\Omega})\right),
$$

satisfying the compatibility condition

$$
\lambda_{1} \operatorname{Trace}\left(\mathfrak{R}_{m_{\epsilon}}\right) \leq \mathfrak{R}_{e_{\epsilon}} \leq \lambda_{2} \operatorname{Trace}\left(\mathfrak{R}_{m_{\epsilon}}\right) \text {, for some } \epsilon \text { independent } \lambda_{1}, \lambda_{2}>0,
$$

such that the following holds:

- Equation of continuity For any $\tau \in(0, T)$ and any $\varphi \in C_{c}^{1}([0, T) \times \bar{\Omega})$ it holds

$$
\left[\int_{\Omega} \varrho_{\epsilon} \varphi \mathrm{d} x\right]_{t=0}^{t=\tau}=\int_{0}^{\tau} \int_{\Omega}\left[\varrho_{\epsilon} \partial_{t} \varphi+\mathbf{m}_{\epsilon} \cdot \nabla_{x} \varphi\right] \mathrm{d} x \mathrm{~d} t ;
$$


- Momentum equation For any $\tau \in(0, T)$ and any $\varphi \in C_{c}^{1}\left([0, T) \times \Omega ; \mathbb{R}^{d}\right)$ with $\left.\boldsymbol{\varphi} \cdot \mathbf{n}\right|_{\partial \Omega}=0$, it holds

$$
\begin{aligned}
& {\left[\int_{\Omega} \mathbf{m}_{\epsilon}(\tau, \cdot) \cdot \boldsymbol{\varphi}(\tau, \cdot) \mathrm{d} x\right]_{t=0}^{t=\tau}} \\
& \quad=\int_{0}^{\tau} \int_{\Omega}\left[\mathbf{m}_{\epsilon} \cdot \partial_{t} \boldsymbol{\varphi}+\left(\frac{\mathbf{m}_{\epsilon} \otimes \mathbf{m}_{\epsilon}}{\varrho_{\epsilon}}\right): \nabla_{x} \boldsymbol{\varphi}+\frac{1}{\epsilon^{2 m}} p\left(\varrho_{\epsilon}\right) \operatorname{div}_{x} \boldsymbol{\varphi}+\frac{1}{\epsilon} \mathbf{b} \times \mathbf{m}_{\epsilon} \cdot \boldsymbol{\varphi}\right] \mathrm{d} x \mathrm{~d} t \\
& \quad+\int_{0}^{\tau} \int_{\Omega} \frac{1}{\epsilon^{2 n}} \varrho_{\epsilon} \nabla_{x} G \cdot \boldsymbol{\varphi} \mathrm{d} x \mathrm{~d} t+\int_{0}^{\tau} \int_{\bar{\Omega}} \nabla_{x} \boldsymbol{\varphi}: \mathrm{d} \Re_{m_{\epsilon}} \mathrm{d} t
\end{aligned}
$$

- Energy inequality The total energy $E_{\epsilon}$ is defined in $[0, T)$ as

$$
E_{\epsilon}(\tau)=\int_{\Omega}\left(\frac{1}{2} \frac{\left|\mathbf{m}_{\epsilon}\right|^{2}}{\varrho_{\epsilon}}+\frac{1}{\epsilon^{2 m}}\left(P\left(\varrho_{\epsilon}\right)-\left(\varrho_{\epsilon}-\tilde{\varrho}_{\epsilon}\right) P^{\prime}\left(\tilde{\varrho}_{\epsilon}\right)\right)-P\left(\tilde{\varrho}_{\epsilon}\right)\right)(\tau, \cdot) \mathrm{d} x .
$$

It satisfies

$$
E_{\epsilon}(\tau)+\int_{\bar{\Omega}} \mathrm{d} \mathfrak{R}_{e_{\epsilon}}(\tau, \cdot) \leq E_{0, \epsilon}
$$

for a.a. $\tau>0$.

Remark 3.4. It is important to define the function

$$
\left(\varrho_{\epsilon}, \mathbf{m}_{\epsilon}\right) \mapsto \frac{1}{2} \frac{\left|\mathbf{m}_{\epsilon}\right|^{2}}{\varrho_{\epsilon}}
$$

on the vacuum set as

$$
\left(\varrho_{\epsilon}, \mathbf{m}_{\epsilon}\right) \mapsto \frac{1}{2} \frac{\left|\mathbf{m}_{\epsilon}\right|^{2}}{\varrho_{\epsilon}}= \begin{cases}\frac{1}{2} \frac{\left|\mathbf{m}_{\epsilon}\right|^{2}}{\varrho_{\epsilon}} & \text { if } \varrho_{\epsilon} \neq 0, \mathbf{m}_{\epsilon} \neq 0, \\ 0 & \text { if } \varrho_{\epsilon}=0, \mathbf{m}_{\epsilon}=0, \\ \infty & \text { if } \varrho_{\epsilon}=0, \mathbf{m}_{\epsilon} \neq 0 .\end{cases}
$$

It follows from the energy inequality (3.6) that for each $\epsilon>0$,

$$
\mathcal{L}^{4}\left(\left\{(t, x) \in(0, T) \times \Omega \mid \frac{1}{2} \frac{\left|\mathbf{m}_{\epsilon}\right|^{2}}{\varrho_{\epsilon}}=\infty\right\}\right)=0,
$$

where $\mathcal{L}^{4}$ is the Lebesgue measure in $\mathbb{R}^{4}$.

In order to study the existence, we first note that the impermeability boundary condition in $\mathbb{R}^{2} \times(0,1)$ can be transformed into periodic ones by considering the space of symmetric functions, see Ebin [15]. Here $\varrho, \mathbf{m}_{h}\left(=m_{1}, m_{2}\right)$ were extended as even functions in the $x_{3}$-variable defined on $\mathbb{R}^{2} \times \mathbb{T}^{1}$, while $m_{3}$ is extended as an odd function in $x_{3}$ on the same set, i.e.,

$$
\begin{aligned}
& \varrho\left(t, x_{h},-x_{3}\right)=\varrho\left(t, x_{h}, x_{3}\right), \mathbf{m}_{h}\left(t, x_{h},-x_{3}\right)=\mathbf{m}_{h}\left(t, x_{h}, x_{3}\right), \\
& m_{3}\left(t, x_{h},-x_{3}\right)=-m_{3}\left(t, x_{h}, x_{3}\right),
\end{aligned}
$$

for all $t \in(0, T), x_{h} \in \mathbb{R}^{2}$ and $x_{3} \in \mathbb{T}^{1}$. A similar convention is adopted for the initial data. Hence, the consideration of the domain $\mathbb{R}^{2} \times(0,1)$ with slip boundary condition is equivalent to $\mathbb{R}^{2} \times \mathbb{T}^{1}$. We have to consider solutions in the class (3.7). The existence of a dissipative solution follows from Breit, Feireisl and Hofmanová as in $[6,7]$. 


\subsection{Relative energy inequality}

In our approach, relative energy functional plays an important role. We consider

$$
\begin{aligned}
\mathcal{E}_{\epsilon}(t) & =\mathcal{E}\left(\varrho_{\epsilon}, \mathbf{m}_{\epsilon} \mid \tilde{\varrho}, \tilde{\mathbf{u}}\right)(t) \\
& :=\int_{\Omega}\left[\frac{1}{2} \varrho_{\epsilon}\left|\frac{\mathbf{m}_{\epsilon}}{\varrho_{\epsilon}}-\tilde{\mathbf{u}}\right|^{2}+\frac{1}{\epsilon^{2 m}}\left(P\left(\varrho_{\epsilon}\right)-P(\tilde{\varrho})-P^{\prime}(\tilde{\varrho})\left(\varrho_{\epsilon}-\tilde{\varrho}\right)\right)\right](t, \cdot) \mathrm{d} x,
\end{aligned}
$$

where $(\tilde{\varrho}, \tilde{\mathbf{u}})$ satisfy

$$
\begin{aligned}
& \left.0<\tilde{\varrho} \in C^{\infty}([0, T]) \times \bar{\Omega}\right) \text { with } \tilde{\varrho}-\tilde{\varrho}_{\epsilon} \text { having compact support in }[0, T] \times \bar{\Omega}, \\
& \tilde{\mathbf{u}} \in C_{c}^{\infty}\left([0, T] \times \bar{\Omega} ; \mathbb{R}^{3}\right) \text { with } \tilde{\mathbf{u}} \cdot \mathbf{n}=0 \text { on } \partial \Omega,
\end{aligned}
$$

and $\left(\varrho_{\epsilon}, \mathbf{m}_{\epsilon}\right)$ is a dissipative solution of the primitive system.

Remark 3.5. The relative energy is a coercive functional (see. Bruell et al. [9] and Březina and Feireisl [12]) satisfying the estimate

$$
\begin{aligned}
\mathcal{E}\left(\varrho_{\epsilon}, \mathbf{u}_{\epsilon} \mid \tilde{\varrho}, \tilde{\mathbf{u}}\right)(t) \geq & \int_{\Omega}\left[\left|\frac{\mathbf{m}_{\epsilon}}{\varrho_{\epsilon}}-\tilde{\mathbf{u}}\right|^{2}\right]_{\mathrm{ess}} \mathrm{d} x+\int_{\Omega}\left[\frac{\left|\mathbf{m}_{\epsilon}\right|^{2}}{\varrho_{\epsilon}}\right]_{\mathrm{res}} \mathrm{d} x \\
& +\frac{1}{\epsilon^{2 m}} \int_{\Omega}\left[\left(\varrho_{\epsilon}-\tilde{\varrho}\right)^{2}\right]_{\mathrm{ess}} \mathrm{d} x+\frac{1}{\epsilon^{2 m}} \int_{\Omega}[1]_{\mathrm{res}}+\left[\varrho_{\epsilon}^{\gamma}\right]_{\mathrm{res}} \mathrm{d} x,
\end{aligned}
$$

where ess and res are considered with respect to $\varrho_{1}=\frac{1}{2} \min _{(0, T) \times \Omega} \tilde{\varrho}$ and $\varrho_{2}=$ $2 \max _{(0, T) \times \Omega} \tilde{\varrho}$.

Using Definition 3.3, from (3.8) we deduce the following relative energy inequality:

$$
\begin{aligned}
\mathcal{E}_{\epsilon}(\tau)+\int_{\bar{\Omega}} \mathrm{d} \Re_{e_{\epsilon}}(\tau, \cdot) \leq & \mathcal{E}_{\epsilon}(0)-\int_{0}^{\tau} \int_{\Omega}\left(\mathbf{m}_{\epsilon}-\varrho_{\epsilon} \tilde{\mathbf{u}}\right) \cdot \partial_{t} \tilde{\mathbf{u}} \mathrm{d} x \mathrm{~d} t \\
& -\int_{0}^{\tau} \int_{\Omega}\left(\frac{\left(\mathbf{m}_{\epsilon}-\varrho_{\epsilon} \tilde{\mathbf{u}}\right) \otimes \mathbf{m}_{\epsilon}}{\varrho_{\epsilon}}\right): \nabla_{x} \tilde{\mathbf{u}} \mathrm{d} x \mathrm{~d} t \\
& -\frac{1}{\epsilon^{2 m}} \int_{0}^{\tau} \int_{\Omega}\left(p\left(\varrho_{\epsilon}\right)-p(\tilde{\varrho})\right) \operatorname{div}_{x} \tilde{\mathbf{u}} \mathrm{d} x \mathrm{~d} t \\
& +\frac{1}{\epsilon^{2 m}} \int_{0}^{\tau} \int_{\Omega}\left(\tilde{\varrho}-\varrho_{\epsilon}\right) \partial_{t} P^{\prime}(\tilde{\varrho}) \mathrm{d} x \mathrm{~d} t \\
& +\frac{1}{\epsilon} \int_{0}^{\tau} \int_{\Omega} \mathbf{b} \times \mathbf{m}_{\epsilon} \cdot \tilde{\mathbf{u}} \mathrm{d} x \mathrm{~d} t \\
& +\frac{1}{\epsilon^{2 m}} \int_{0}^{\tau} \int_{\Omega}\left(\tilde{\varrho} \tilde{\mathbf{u}}-\mathbf{m}_{\epsilon}\right) \cdot\left(\nabla_{x} P^{\prime}(\tilde{\varrho})-\nabla_{x} P^{\prime}(\tilde{\varrho} \epsilon)\right) \mathrm{d} x \mathrm{~d} t \\
& -\frac{1}{\epsilon^{2 n}} \int_{0}^{\tau} \int_{\Omega}\left(\varrho_{\epsilon}-\tilde{\varrho}\right) \nabla_{x} G \cdot \tilde{\mathbf{u}} \mathrm{d} x \mathrm{~d} t \\
& -\int_{0}^{\tau} \int_{\Omega} \nabla_{x} \tilde{\mathbf{u}}: \mathrm{d} \Re_{m_{\epsilon}}(t, \cdot) \mathrm{d} t .
\end{aligned}
$$




\subsubsection{A possible adaptation for Sobolev test functions}

From Remark 3.5, it is clear that the relative energy functional is nonnegative and if $\mathcal{E}_{\epsilon} \rightarrow 0$ as $\epsilon \rightarrow 0$ we obtain some strong local convergence of the state variables. So we want to substitute $(\tilde{\varrho}, \tilde{u})$ suitably by the solutions of target system. We notice that the existence of strong solutions is known in the space of Sobolev functions, see Propositions 3.1 and 3.2. Therefore, following [5] we can extend the inequality (3.10) for the test functions $(\tilde{\varrho}, \tilde{\mathbf{u}})$ having Sobolev regularities, i.e., $\left(\tilde{\varrho}-\tilde{\varrho}_{\epsilon}, \tilde{\mathbf{u}}\right) \in$ $C^{1}\left([0, T] ; W^{k, 2}(\Omega)\right) \times C^{1}\left([0, T] ; W^{k, 2}\left(\Omega ; \mathbb{R}^{3}\right)\right)$ with $k \geq 3$ and $\tilde{\mathbf{u}} \cdot \mathbf{n}=0$ on $\partial \Omega$.

\section{Main results and their proofs}

First we state our main results for both cases:

4.1. Main theorem: Case I

\subsubsection{Properties of a static solution}

First, we notice that a static solution $\left(\tilde{\varrho}_{\epsilon}, \mathbf{0}\right)$ satisfies

$$
\nabla_{x} p\left(\tilde{\varrho}_{\epsilon}\right)=\epsilon^{2(m-n)} \tilde{\varrho}_{\epsilon} \nabla_{x} G .
$$

In terms of the pressure potential, we rewrite the above equation as

$$
\nabla_{x} P^{\prime}\left(\tilde{\varrho}_{\epsilon}\right)=\epsilon^{2(m-n)} \nabla_{x} G .
$$

So, we obtain

$$
P^{\prime}\left(\tilde{\varrho}_{\epsilon}\right)=-\epsilon^{2(m-n)} x_{3}+C,
$$

where $C$ is a constant. As a consequence of $G=\left(0,0,-x_{3}\right)$, we have $\tilde{\varrho}_{\epsilon}(x)=\tilde{\varrho}_{\epsilon}\left(x_{3}\right)$. Without loss of generality, we consider $C=1$. We know that $P^{\prime}(s) \approx s^{\gamma-1}$ for $s \geq 0$. To reduce complication, here we assume $P^{\prime}(s)=s^{\gamma-1}$, for $s \geq 0$. We also have

$$
P^{\prime \prime}\left(\tilde{\varrho}_{\epsilon}\right) \nabla_{x} \tilde{\varrho}_{\epsilon}=\epsilon^{2(m-n)} .
$$

For $0<\epsilon<\frac{1}{2}$, we observe that a static solution $\tilde{\varrho}_{\epsilon}$ satisfies the following property:

$$
\begin{aligned}
& 0<\tilde{\varrho}_{\epsilon} \in C^{\infty}([0,1]), \\
& \sup _{x_{3} \in[0,1]}\left|\tilde{\varrho}_{\epsilon}\left(x_{3}\right)-1\right| \leq \epsilon^{\frac{2(m-n)}{\gamma-1}}, \sup _{x_{3} \in[0,1]}\left|\nabla_{x} \tilde{\varrho}_{\epsilon}\left(x_{3}\right)\right| \leq \epsilon^{2(m-n)} .
\end{aligned}
$$

Remark 4.1. Since, we are interested for the case $\epsilon \rightarrow 0$, the consideration of $0<$ $\epsilon<\frac{1}{2}$ is justified. Furthermore, if $\gamma>2$ and $\epsilon<1$ we have

$$
\sup _{x_{3} \in[0,1]}\left|\tilde{\varrho}_{\epsilon}\left(x_{3}\right)-1\right| \leq \epsilon^{2(m-n)} .
$$

As $m>n$, asymptotically, the static solution approaches the constant state $\tilde{\varrho}=1$ as $\epsilon \rightarrow 0$. 


\subsubsection{Well-prepared data}

We say that the set of initial data $\left\{\left(\varrho_{0, \epsilon}, \mathbf{m}_{0, \epsilon}\right)\right\}_{\epsilon>0}$ is well-prepared if

$$
\begin{aligned}
& \varrho_{\epsilon}(0, \cdot)=\varrho_{0, \epsilon}=\tilde{\varrho_{\epsilon}}+\epsilon^{m} \varrho_{0, \epsilon}^{(1)} \text { with }\left\{\varrho_{0, \epsilon}^{(1)}\right\}_{\epsilon>0} \text { is bounded in } L^{2} \cap L^{\infty}(\Omega) \\
& \text { and } \varrho_{0, \epsilon}^{(1)} \rightarrow 0 \text { in } L^{2}(\Omega), \\
& \mathbf{u}_{0, \epsilon}=\frac{\mathbf{m}_{0, \epsilon}}{\varrho_{0, \epsilon}} \rightarrow \mathbf{v}_{0}=\left(\mathbf{v}_{0}^{(1)}, \mathbf{v}_{0}^{(2)}, 0\right) \text { in } L^{2}\left(\Omega ; \mathbb{R}^{3}\right) \text { with } \operatorname{div}_{x_{h}} \mathbf{v}_{0}=0
\end{aligned}
$$

We provide the main result for this case.

Theorem 4.2. Let $\left(\varrho_{\epsilon}, \mathbf{m}_{\epsilon}\right)$ be a dissipative solution of the system (1.1)-(1.7) with $\frac{m}{2}>n \geq 1$. Moreover, we assume that the initial data are well-prepared, i.e., it satisfies (4.3) and $\mathbf{v}_{0} \in W^{k, 2}\left(\mathbb{R}^{2}\right)$ with $k \geq 3$. Then,

$$
\begin{aligned}
& \text { ess } \sup _{t \in(0, T)}\left\|\varrho_{\epsilon}-\tilde{\varrho}_{\epsilon}\right\|_{\left(L^{2}+L^{\gamma^{\prime}}\right)(\Omega)} \leq \epsilon^{m} c, \\
& \frac{\mathbf{m}_{\epsilon}}{\sqrt{\varrho_{\epsilon}}} \rightarrow \mathbf{v}\left\{\begin{array}{l}
\text { weak- }(*) \text { ly in } L^{\infty}\left(0, T ; L^{2}\left(\Omega ; \mathbb{R}^{3}\right)\right), \\
\text { strongly in } L_{l o c}^{1}\left((0, T) \times \Omega ; \mathbb{R}^{3}\right),
\end{array}\right.
\end{aligned}
$$

where $c>0, \gamma^{\prime}=\min \{2, \gamma\}$ and $\mathbf{v}=\left(\mathbf{v}_{h}, 0\right)$ is the unique solution of the incompressible Euler system with initial data $\mathbf{v}_{0}$ in $\mathbb{R}^{2}$.

\subsection{Main theorem: Case II}

\subsubsection{Well-prepared data}

Let $\hat{\varrho}$ be a static solution satisfying (3.1). We say that the set of initial data $\left(\varrho_{0, \epsilon}\right.$, $\left.\mathbf{m}_{0, \epsilon}\right)_{(\epsilon>0)}$ is well-prepared if

$$
\begin{aligned}
& \varrho_{0, \epsilon}=\hat{\varrho}+\epsilon \varrho_{0, \epsilon}^{(1)},\left\{\varrho_{0, \epsilon}\right\}_{\{\epsilon>0\}} \text { is bounded in } L^{2} \cap L^{\infty}(\Omega), \varrho_{0, \epsilon}^{(1)} \rightarrow q_{0} \text { in } L^{2}(\Omega), \\
& \frac{\mathbf{m}_{0, \epsilon}}{\varrho_{0, \epsilon}} \rightarrow \mathbf{v}_{0}=\left(\mathbf{v}_{0}^{(1)}, \mathbf{v}_{0}^{(2)}, 0\right) \text { in } L^{2}\left(\Omega ; \mathbb{R}^{3}\right) \text { with the relation } \\
& -\Delta_{x_{h}} q_{0}=\hat{\varrho} \operatorname{curl}_{x_{h}} P_{h}\left(\mathbf{v}_{0}\right), \text { and } q_{0} \in L^{2}\left(\mathbb{R}^{2}\right) .
\end{aligned}
$$

Theorem 4.3. Let $\left(\varrho_{\epsilon}, \mathbf{m}_{\epsilon}\right)$ be a dissipative solution of the system (1.1)-(1.7) with $m=n=1$. Moreover, we assume that the initial data are well-prepared, i.e., it satisfies (4.3) and $q_{0} \in W^{k, 2}$ with $k \geq 4$. Let $(q, \mathbf{v})$ solves (2.4) for initial data $q_{0}$. Then after taking a subsequence, the following holds

$$
\begin{aligned}
& \varrho_{\epsilon}^{(1)} \equiv \frac{\varrho_{\epsilon}-\hat{\varrho}}{\epsilon} \rightarrow q \text { weak- }(*) \text { ly in } L^{\infty}\left(0, T ; L^{2}+L^{\gamma^{\prime}}(\Omega)\right), \\
& \mathbf{m}_{\epsilon} \rightarrow \hat{\varrho} \mathbf{v} \text { weak- }(*) \text { ly in } L^{\infty}\left(0, T ; L^{2}+L^{\frac{2 \gamma}{\gamma+1}}(\Omega)\right),
\end{aligned}
$$


where $\gamma^{\prime}=\min \{2, \gamma\}$. Furthermore, we have

$$
\frac{\mathbf{m}_{\epsilon}}{\sqrt{\varrho_{\epsilon}}} \rightarrow \mathbf{v} \text { strongly in } L_{l o c}^{1}\left((0, T) \times \Omega ; \mathbb{R}^{3}\right) .
$$

Remark 4.4. In both cases, we note that the choice of well-prepared initial data implies (3.4), hence the consideration of the dissipative solution of the primitive system is justified.

In the following subsections, we give the proofs.

\subsection{Proof of Theorem 4.2}

\subsubsection{Uniform bound and weak convergence}

First, we note that $\tilde{\mathbf{u}}=0$ and $\tilde{\varrho}=\tilde{\varrho}_{\epsilon}$ satisfy (3.9). Hence, we use them as test functions in the relative energy inequality (3.10). One the other hand, the choice of (4.2) ensures that the initial energy $E_{0, \epsilon}$ is uniformly bounded. Thus, we have the following bounds

$$
\begin{aligned}
& \text { ess } \sup _{t \in(0, T)}\left\|\frac{\mathbf{m}_{\epsilon}}{\sqrt{\varrho_{\epsilon}}}\right\|_{L^{2}\left(\Omega ; \mathbb{R}^{3}\right)} \leq C, \\
& \text { ess } \sup _{t \in(0, T)}\left\|\left[\frac{\varrho_{\epsilon}-\tilde{\varrho}_{\epsilon}}{\epsilon^{m}}\right]_{\mathrm{ess}}\right\|_{L^{2}(\Omega)} \leq C, \\
& \text { ess } \sup _{t \in(0, T)}\left\|\left[\varrho_{\epsilon}\right]_{\mathrm{res}}\right\|_{L^{\gamma}(\Omega)}^{\gamma}+\text { ess } \sup _{t \in(0, T)}\left\|[1]_{\mathrm{res}}\right\|_{L^{\gamma}(\Omega)}^{\gamma} \leq \epsilon^{2 m} C,
\end{aligned}
$$

where $C$ is independent of $\epsilon$. We consider $\gamma^{\prime}=\min \{2, \gamma\}$. Estimate (4.4) and the fact $\gamma^{\prime} \leq 2$ imply

$$
\text { ess } \sup _{t \in(0, T)}\left\|\varrho_{\epsilon}-\tilde{\varrho}_{\epsilon}\right\|_{\left(L^{2}+L^{\gamma^{\prime}}\right)(\Omega)} \leq\left(\epsilon^{m}+\epsilon^{\frac{2 m}{\gamma^{\prime}}}\right) C \leq \epsilon^{m} C .
$$

Equation (4.5), together with (4.1), yields

$$
\varrho_{\epsilon} \rightarrow 1 \text { in } L^{\infty}\left(0, T ; L_{\text {loc }}^{q}(\Omega)\right) \text { for any } 1 \leq q<\gamma^{\prime} .
$$

Also, from the uniform bound (4.4) and (4.6) imply that

$$
\frac{\mathbf{m}_{\epsilon}}{\sqrt{\varrho_{\epsilon}}} \rightarrow \text { u weak-(*)ly in } L^{\infty}\left(0, T ; L^{2}\left(\Omega ; \mathbb{R}^{3}\right)\right),
$$

and

$$
\mathbf{m}_{\epsilon} \rightarrow \mathbf{m} \text { weak-(*)lyin } L^{\infty}\left(0, T ; L^{2}+L^{\grave{\gamma}}\left(\Omega ; \mathbb{R}^{3}\right)\right),
$$

passing to suitable subsequence, where $\dot{\gamma}=\min \left\{\frac{4}{3}, \frac{2 \gamma}{\gamma+1}\right\}$. The strong convergence of the density (4.6) helps to obtain $\mathbf{m}=\mathbf{u}$ in the weak sense.

Finally, we may let $\epsilon \rightarrow 0$ in the continuity equation to deduce that,

$$
\int_{0}^{\tau} \int_{\Omega} \mathbf{u} \cdot \nabla_{x} \varphi \mathrm{d} x \mathrm{~d} t=0, \forall \varphi \in C_{c}^{\infty}(\Omega)
$$




\subsubsection{Strong convergence}

Here we choose proper test functions and prove that $\lim _{\epsilon \rightarrow 0} \mathcal{E}_{\epsilon}(t)=0$.

Taking motivation from (2.1), we consider another equation that describes a nonoscillatory part described by a variable $q_{\epsilon}$, that satisfies

$$
\partial_{t}\left(\Delta_{x_{h}} q_{\epsilon}-\left(\epsilon^{m-1}\right)^{2} q_{\epsilon}\right)+\frac{1}{\epsilon^{m-1}} \nabla_{x_{h}}^{\perp} q_{\epsilon} \cdot \nabla_{x_{h}}\left(\Delta_{x_{h}} q_{\epsilon}-\left(\epsilon^{m-1}\right)^{2} q_{\epsilon}\right)=0,
$$

in $\mathbb{R}^{2}$ supplemented with initial data $q_{\epsilon}(0, \cdot)=q_{0, \epsilon}$ such that

$$
-\Delta_{x_{h}} q_{0, \epsilon}+\left(\epsilon^{2(m-1)}\right) q_{0, \epsilon}=\epsilon^{m-1} \operatorname{curl}_{x_{h}} P_{h}\left(\mathbf{v}_{0}\right)
$$

Let us introduce another variable $\mathbf{v}_{\epsilon}$ such that $\mathbf{v}_{\epsilon}$ and $q_{\epsilon}$ are interrelated by

$$
\nabla_{x} q_{\epsilon}+\epsilon^{m-1} \mathbf{b} \times \mathbf{v}_{\epsilon}=0 .
$$

Thus, initial data for $\mathbf{v}_{\epsilon}$ satisfy

$$
-\nabla_{x_{h}} q_{0, \epsilon}=\epsilon^{m-1} \mathbf{b} \times \mathbf{v}_{0, \epsilon} .
$$

From the hypothesis on initial data in Theorem 4.2, we have

$$
\mathbf{v}_{0} \in W^{k, 2}\left(\mathbb{R}^{2}\right) \text { with } k \geq 3
$$

We observe that $\left\|q_{0, \epsilon}\right\|_{L^{2}\left(\mathbb{R}^{2}\right)} \leq C$ and $\left\|\nabla_{x} q_{0, \epsilon}\right\|_{L^{2}\left(\mathbb{R}^{2}\right)} \leq \epsilon^{m-1} C$. Therefore, we can consider $\left\{q_{0, \epsilon}\right\}_{\epsilon>0}$ such that $q_{0, \epsilon} \rightarrow 0$ in $L^{2}\left(\mathbb{R}^{2}\right)$ as $\epsilon \rightarrow 0$. Furthermore, we also note that $\mathbf{v}_{0, \epsilon} \rightarrow P_{h}\left(\mathbf{v}_{0}\right)$ as $\epsilon \rightarrow 0$.

In order to have a simplified notation, we consider $\omega=\epsilon^{m-1}$ and $\tilde{q}_{\epsilon}=\frac{q_{\epsilon}}{\omega}$. We rewrite (4.7) as

$$
\partial_{t}\left(\Delta_{x_{h}} \tilde{q}_{\epsilon}-\omega^{2} \tilde{q}_{\epsilon}\right)+\nabla_{x_{h}}^{\perp} \tilde{q}_{\epsilon} \cdot \nabla_{x_{h}}\left(\Delta_{x_{h}} \tilde{q}_{\epsilon}-\omega^{2} q_{\epsilon}\right)=0,
$$

We notice that Eq. (4.9) has a similar structure to that of the geophysical flows. Thus, we apply Oliver [33, Theorem 3] to ensure the existence and uniqueness of solution $\tilde{q}_{\epsilon}$.

In order to obtain a uniform estimate independent of $\epsilon$, we multiply the (4.9) by $q_{\epsilon}$ and performing integration by parts, we get

$$
\int_{\mathbb{R}^{2}}\left(\left|\nabla_{x_{h}} \tilde{q}_{\epsilon}\right|^{2}+\omega^{2}\left|\tilde{q}_{\epsilon}\right|^{2}\right)(t, \cdot) \mathrm{d} x=\int_{\mathbb{R}^{2}}\left(\left|\nabla_{x_{h}} \tilde{q}_{0, \epsilon}\right|^{2}+\omega^{2}\left|\tilde{q}_{0, \epsilon}\right|^{2}\right) \mathrm{d} x,
$$

for a.e. $t \in(0, T)$. As the initial data for $\tilde{q}_{\epsilon}$ depends only on $\mathbf{v}_{0}$, we deduce that

$$
\begin{aligned}
\left\{-\Delta_{x_{h}} \tilde{q}_{\epsilon}\right. & \left.+\omega^{2} \tilde{q}_{\epsilon}\right\}_{\epsilon>0} \text { is bounded in } C^{1}\left([0, T] ; W^{k-2,2}\left(\mathbb{R}^{2}\right)\right) \\
& \cap C\left([0, T] ; W^{k-1,2}\left(\mathbb{R}^{2}\right)\right) .
\end{aligned}
$$


Now, from (4.8), we also obtain

$$
\left\{\mathbf{v}_{\epsilon}\right\}_{\epsilon>0} \text { is bounded in } C\left([0, T] ; W^{k, 2}\left(\mathbb{R}^{2}\right)\right) \cap C^{1}\left([0, T] ; W^{k-1,2}\left(\mathbb{R}^{2}\right)\right) .
$$

It is easy to verify that $\partial_{t} \tilde{q}_{\epsilon}$ satisfies the equation

$$
\partial_{t} q_{\epsilon}=\left(\Delta_{x_{h}}-\omega^{2}\right)^{-1}\left(\mathbf{v}_{\epsilon} \operatorname{curl}_{x_{h}} \mathbf{v}_{\epsilon}\right) .
$$

Consequently, it yields

$$
\left\{\partial_{t} \tilde{q}_{\epsilon}\right\}_{\epsilon>0} \text { is bounded in } C\left([0, T] ; W^{k-1,2}\left(\mathbb{R}^{2}\right)\right),
$$

and

$$
\left\{\partial_{t} \mathbf{v}_{\epsilon}\right\}_{\epsilon>0} \text { is bounded in } C\left([0, T] ; W^{k, 2}\left(\mathbb{R}^{2} ; \mathbb{R}^{2}\right)\right) \text {. }
$$

This bounds are independent of $\epsilon$.

Therefore, we obtain the following weak convergence:

$$
\mathbf{v}_{\epsilon} \rightarrow \mathbf{v} \text { weakly in } C\left([0, T] ; W^{k, 2}\left(\mathbb{R}^{2}\right)\right),
$$

and

$$
\partial_{t} \mathbf{v}_{\epsilon} \rightarrow \partial_{t} \mathbf{v} \text { weakly in } C\left([0, T] ; W^{k-1,2}\left(\mathbb{R}^{2}\right)\right)
$$

Since $k \geq 4$, applying Sobolev embedding theorem, we obtain

$$
\mathbf{v}_{\epsilon} \rightarrow \mathbf{v} \text { in } L^{q}\left(0, T ; L_{\mathrm{loc}}^{q}\left(\mathbb{R}^{2}\right)\right) .
$$

We rewrite (4.9) as

$$
\partial_{t}\left(\operatorname{curl}_{x_{h}} \mathbf{v}_{\epsilon}\right)-\omega^{2} \partial_{t} \tilde{q}_{\epsilon}+\mathbf{v}_{\epsilon} \nabla_{x}\left(\operatorname{curl}_{x_{h}} \mathbf{v}_{\epsilon}\right)=0 .
$$

From (4.11), we infer that

$$
\partial_{t}\left(\operatorname{curl}_{x_{h}} \mathbf{v}\right)+\mathbf{v} \cdot \nabla_{x}\left(\operatorname{curl}_{x_{h}} \mathbf{v}\right)=0 .
$$

This is similar to (2.2). We also have

$$
\Pi \in C\left([0, T] ; W^{k, 2}\left(\mathbb{R}^{2}\right)\right) .
$$

Clearly we have the following estimates

$$
\begin{aligned}
& \left\|q_{\epsilon}\right\|_{L^{\infty}\left(0, T ; L^{2}(\Omega)\right)} \leq C, \\
& \left\|\partial_{t} q_{\epsilon}\right\|_{L^{\infty}\left(0, T ; L^{q}(\Omega)\right)}+\left\|\nabla_{x_{h}} q_{\epsilon}\right\|_{L^{\infty}\left(0, T ; L^{2}(\Omega)\right)} \leq \epsilon^{m-1} C,
\end{aligned}
$$

for $q \geq 2$. Also $\mathbf{v}_{\epsilon} \in C\left([0, T] ; W^{k-1,2}\left(\mathbb{R}^{2}\right)\right)$ with $k \geq 4$ implies

$$
\left\|q_{\epsilon}\right\|_{L^{\infty}\left((0, T) \times \mathbb{R}^{2}\right)} \leq C .
$$


Now, we consider a suitable test function for the relative energy inequality(3.10) as

$$
\tilde{\mathbf{u}}=\mathbf{V}_{\epsilon}=\left(\mathbf{v}_{\epsilon}, 0\right), \tilde{\varrho}=\tilde{\varrho}_{\epsilon}+\epsilon^{m} q_{\epsilon},
$$

where $\left(q_{\epsilon}, \mathbf{v}_{3}\right)$ satisfies (4.7) ans (4.8) and $\tilde{\varrho}_{\epsilon}$ is a static solution that satisfies (4.1). We use the relation between $q_{\epsilon}$ and $\mathbf{v}_{\epsilon}$ and obtain

$$
\begin{aligned}
& \mathcal{E}_{\epsilon}(\tau)+\int_{\bar{\Omega}} \mathrm{d} \Re_{e_{\epsilon}}(\tau, \cdot) \leq \mathcal{E}_{\epsilon}(0) \\
& -\int_{0}^{\tau} \int_{\Omega}\left(\mathbf{m}_{\epsilon}-\varrho_{\epsilon} \mathbf{V}_{\epsilon}\right) \cdot\left(\partial_{t} \mathbf{V}_{\epsilon}+\left(\mathbf{V}_{\epsilon} \cdot \nabla_{x}\right) \mathbf{V}_{\epsilon}\right) \mathrm{d} x \mathrm{~d} t \\
& -\int_{0}^{\tau} \int_{\Omega}\left(\frac{\left(\mathbf{m}_{\epsilon}-\varrho_{\epsilon} \mathbf{V}_{\epsilon}\right) \otimes\left(\mathbf{m}_{\epsilon}-\varrho_{\epsilon} \mathbf{V}_{\epsilon}\right)}{\varrho_{\epsilon}}\right): \nabla_{x} \mathbf{V}_{\epsilon} \mathrm{d} x \mathrm{~d} t \\
& +\frac{1}{\epsilon^{2 m}} \int_{0}^{\tau} \int_{\Omega}\left(\tilde{\varrho}-\varrho_{\epsilon}\right) \partial_{t} P^{\prime}(\tilde{\varrho}) \mathrm{d} x \mathrm{~d} t \\
& +\frac{1}{\epsilon^{m}} \int_{0}^{\tau} \int_{\Omega} \mathbf{m}_{\epsilon} \cdot \nabla_{x} q_{\epsilon}\left(P^{\prime \prime}(\tilde{\varrho})-P^{\prime \prime}(1)\right) \mathrm{d} x \mathrm{~d} t \\
& +\frac{1}{\epsilon^{2 m}} \int_{0}^{\tau} \int_{\Omega} \mathbf{m}_{\epsilon} \cdot\left(P^{\prime \prime}(\tilde{\varrho})-P^{\prime \prime}\left(\tilde{\varrho_{\epsilon}}\right)\right) \nabla_{x} \tilde{\varrho}_{\epsilon} \mathrm{d} x \mathrm{~d} t \\
& -\frac{1}{\epsilon^{2 n}} \int_{0}^{\tau} \int_{\Omega}\left(\varrho_{\epsilon}-\tilde{\varrho}\right) \nabla_{x} G \cdot \mathbf{V}_{\epsilon} \mathrm{d} x \mathrm{~d} t \\
& -\int_{0}^{\tau} \int_{\bar{\Omega}} \nabla_{x} \tilde{\mathbf{u}}: \mathrm{d} \Re_{m_{\epsilon}}(t, \cdot) \mathrm{d} t=\Sigma_{i=1}^{8} \mathcal{L}_{i} .
\end{aligned}
$$

Here we compute each term $\mathcal{L}_{i}, i=1, \cdots, 8$ of (4.14). For term $\mathcal{L}_{1}$, we have

$$
\mathcal{E}_{\epsilon}\left(\varrho_{0, \epsilon},(\varrho \mathbf{u})_{0, \epsilon} \mid \tilde{\varrho}_{\epsilon}+\epsilon^{m} q_{0, \epsilon}, \mathbf{v}_{0}\right) \leq\left\|\frac{(\varrho \mathbf{u})_{0, \epsilon}}{\varrho_{0, \epsilon}}-\mathbf{v}_{0}\right\|_{L^{2}(\Omega)}^{2}+\left\|\varrho_{0, \epsilon}^{(1)}-q_{0, \epsilon}\right\|_{L^{2}(\Omega)}^{2} .
$$

Consideration of well-prepared data yields

$$
\left|\mathcal{L}_{1}\right| \leq \xi(\epsilon) .
$$

From now on, we use this generic function $\xi(\cdot)$, such that $\lim _{\epsilon \rightarrow 0} \xi(\epsilon)=0$.

We rewrite $\mathcal{L}_{2}$ as

$$
\begin{aligned}
\mathcal{L}_{2}= & -\int_{0}^{\tau} \int_{\Omega}\left(\mathbf{m}_{\epsilon}-\varrho_{\epsilon} \mathbf{V}_{\epsilon}\right) \cdot\left(\partial_{t} \mathbf{V}_{\epsilon}+\left(\mathbf{V}_{\epsilon} \cdot \nabla_{x}\right) \mathbf{V}_{\epsilon}\right) \mathrm{d} x \mathrm{~d} t \\
= & -\int_{0}^{\tau} \int_{\Omega} \mathbf{m}_{\epsilon} \cdot\left(\partial_{t} \mathbf{V}_{\epsilon}+\left(\mathbf{V}_{\epsilon} \cdot \nabla_{x}\right) \mathbf{V}_{\epsilon}\right) \mathrm{d} x \mathrm{~d} t \\
& +\int_{0}^{\tau} \int_{\Omega}\left(\varrho_{\epsilon}-1\right) \mathbf{V}_{\epsilon} \cdot \partial_{t} \mathbf{V}_{\epsilon} \mathrm{d} x \mathrm{~d} t+\int_{0}^{\tau} \int_{\Omega} \partial_{t} \mathbf{V}_{\epsilon} \cdot \mathbf{V}_{\epsilon} \mathrm{d} x \mathrm{~d} t \\
= & \mathcal{L}_{2,1}+\mathcal{L}_{2,2}+\mathcal{L}_{2,3} .
\end{aligned}
$$

Using (4.12) and (4.1), we obtain

$$
\left|\mathcal{L}_{2,2}\right| \leq \xi(\epsilon)
$$


We claim

$$
\mathcal{L}_{2,1} \rightarrow-\int_{0}^{\tau} \int_{\Omega} \mathbf{u} \cdot \nabla_{x_{h}} \Pi \mathrm{d} x \mathrm{~d} t=0
$$

as $\epsilon \rightarrow 0$. Let, $K$ be a compact subset of $\mathbb{R}^{2}$. We use (4.11) to deduce

$$
\begin{aligned}
\int_{0}^{\tau} & \int_{K \times(0,1)} \mathbf{m}_{\epsilon} \cdot\left(\partial_{t} \mathbf{V}_{\epsilon}+\left(\mathbf{V}_{\epsilon} \cdot \nabla_{x}\right) \mathbf{V}_{\epsilon}\right) \mathrm{d} x \mathrm{~d} t \\
& \rightarrow \int_{0}^{\tau} \int_{K \times(0,1)} \mathbf{u} \cdot\left(\partial_{t} \mathbf{v}+\left(\mathbf{v} \cdot \nabla_{x}\right) \mathbf{v}\right) \mathrm{d} x \mathrm{~d} t
\end{aligned}
$$

where $\mathbf{v}=\left(\mathbf{v}_{h}, 0\right)$. Using the fact that $\Pi \in C\left([0, T] ; W^{k, 2}\left(\mathbb{R}^{2}\right)\right)$ with $k \geq 3$, we have

$$
\left|\mathcal{L}_{2,1}\right| \leq \xi(\epsilon)
$$

We want to estimate the term $\mathcal{L}_{4}$. First, we rewrite it as

$$
\begin{aligned}
\mathcal{L}_{4}= & \frac{1}{\epsilon^{m}} \int_{0}^{\tau} \int_{\Omega}\left(\tilde{\varrho}-\varrho_{\epsilon}\right) P^{\prime \prime}(\tilde{\varrho}) \partial_{t} q_{\epsilon} \mathrm{d} x \mathrm{~d} t \\
= & \int_{0}^{\tau} \int_{\Omega}\left(P^{\prime \prime}(\tilde{\varrho})-P^{\prime \prime}\left(\tilde{\varrho}_{\epsilon}\right)\right)\left(q_{\epsilon}-\frac{\varrho_{\epsilon}-\tilde{\varrho}_{\epsilon}}{\epsilon^{m}}\right) \partial_{t} q_{\epsilon} \mathrm{d} x \mathrm{~d} t \\
& +\int_{0}^{\tau} \int_{\Omega}\left(P^{\prime \prime}\left(\tilde{\varrho}_{\epsilon}\right)-P^{\prime \prime}(1)\right)\left(q_{\epsilon}-\frac{\varrho_{\epsilon}-\tilde{\varrho}_{\epsilon}}{\epsilon^{m}}\right) \partial_{t} q_{\epsilon} \mathrm{d} x \mathrm{~d} t \\
& +\int_{0}^{\tau} \int_{\Omega} q_{\epsilon} \partial_{t} q_{\epsilon} \mathrm{d} x \mathrm{~d} t-\int_{0}^{\tau} \int_{\Omega} \frac{\varrho_{\epsilon}-\tilde{\varrho}_{\epsilon}}{\epsilon^{m}} \partial_{t} q_{\epsilon} \mathrm{d} x \mathrm{~d} t \\
= & \mathcal{L}_{4,1}+\mathcal{L}_{4,2}+\mathcal{L}_{4,3}+\mathcal{L}_{4,4} .
\end{aligned}
$$

We note that, for each $x \in \Omega$ we get

$$
\left(P^{\prime \prime}(\tilde{\varrho})-P^{\prime \prime}\left(\tilde{\varrho}_{\epsilon}\right)\right) \leq C \epsilon^{m}\left|q_{\epsilon}(x)\right| .
$$

Using the above inequality and (4.12), we deduce that

$$
\begin{aligned}
\left|\mathcal{L}_{4,1}\right| \leq \epsilon^{m} \sup _{t \in(0, T)}\left\|\left[\frac{\varrho_{\epsilon}-\tilde{\varrho}_{\epsilon}}{\epsilon^{m}}\right]_{\mathrm{ess}}-q_{\epsilon}\right\|_{L^{2}(\Omega)}\left\|\partial_{t} q_{\epsilon}\right\|_{L^{\infty}([0, T] \times \Omega)}\left\|q_{\epsilon}\right\|_{L^{\infty}\left(0, T ; L^{2}(\Omega)\right)} \\
+\epsilon^{m} \sup _{t \in(0, T)}\left\|\left[\frac{\varrho_{\epsilon}-\tilde{\varrho}_{\epsilon}}{\epsilon^{m}}\right]_{\operatorname{res}}\right\|_{L^{\gamma^{\prime}(\Omega)}}\left\|q_{\epsilon}\right\|_{L^{\infty}\left(0, T ; L^{\prime} \gamma_{(\Omega)}\right)}\left\|\partial_{t} q_{\epsilon}\right\|_{L^{\infty}([0, T] \times \Omega)},
\end{aligned}
$$

where $\frac{1}{\gamma^{\prime}}+\frac{1}{\gamma^{\prime *}}=1$.

Similarly, using (4.1) we have 


$$
\begin{aligned}
\left|\mathcal{L}_{4,2}\right| \leq & \epsilon^{\frac{2(m-n)}{\gamma-1}} \sup _{t \in(0, T)}\left\|\left[\frac{\varrho_{\epsilon}-\tilde{\varrho}_{\epsilon}}{\epsilon^{m}}\right]_{\mathrm{ess}}-q_{\epsilon}\right\|_{L^{2}(\Omega)}\left\|\partial_{t} q_{\epsilon}\right\|_{L^{\infty}\left(0, T ; L^{2}(\Omega)\right)} \\
& +\epsilon^{\frac{2(m-n)}{\gamma-1}} \sup _{t \in(0, T)}\left\|\left[\frac{\varrho_{\epsilon}-\tilde{\varrho}_{\epsilon}}{\epsilon^{m}}\right]_{\mathrm{res}}\right\|_{L^{\gamma^{\prime}(\Omega)}}\left\|\partial q_{\epsilon}\right\|_{L^{\infty}\left(0, T ; L^{\gamma^{\prime *}}(\Omega)\right)},
\end{aligned}
$$

for $1<\gamma \leq 2$, and

$$
\begin{aligned}
\left|\mathcal{L}_{4,2}\right| \leq & \epsilon^{2(m-n)} \sup _{t \in(0, T)}\left\|\left[\frac{\varrho_{\epsilon}-\tilde{\varrho}_{\epsilon}}{\epsilon^{m}}\right]_{\mathrm{ess}}-q_{\epsilon}\right\|_{L^{2}(\Omega)}\left\|\partial_{t} q_{\epsilon}\right\|_{L^{\infty}\left(0, T ; L^{2}(\Omega)\right)} \\
& +\epsilon^{2(m-n)} \sup _{t \in(0, T)}\left\|\left[\frac{\varrho_{\epsilon}-\tilde{\varrho}_{\epsilon}}{\epsilon^{m}}\right]_{\operatorname{res}}\right\|_{L^{\gamma^{\prime}(\Omega)}}\left\|\partial q_{\epsilon}\right\|_{L^{\infty}\left(0, T ; L^{\gamma^{\prime *}}(\Omega)\right)},
\end{aligned}
$$

for $\gamma>2$.

Analogously, we deduce

$$
\left|\mathcal{L}_{4,4}\right| \leq\left\|\frac{\varrho_{\epsilon}-\tilde{\varrho}_{\epsilon}}{\epsilon^{m}}\right\|_{L^{\infty}\left(0, T ; L^{2}+L^{\gamma^{\prime}}(\Omega)\right)}\left\|\partial_{t} q_{\epsilon}\right\|_{L^{\infty}\left(0, T ; L^{2} \cap L^{\gamma^{\prime *}}(\Omega)\right)},
$$

where $\frac{1}{\gamma^{\prime}}+\frac{1}{\gamma^{\prime *}}=1$. We use estimate (4.12) to conclude

$$
\left|\mathcal{L}_{4,1}\right|+\left|\mathcal{L}_{4,2}\right|+\left|\mathcal{L}_{4,4}\right| \leq \xi(\epsilon)
$$

The equation (4.10) implies

$$
\mathcal{L}_{2,3}+\mathcal{L}_{4,3}=\int_{0}^{\tau} \int_{\Omega}\left(\partial_{t} \mathbf{v}_{\epsilon} \cdot \mathbf{v}_{\epsilon}+q_{\epsilon} \partial_{t} q_{\epsilon}\right) \mathrm{d} x \mathrm{~d} t=0 .
$$

Therefore, combining all estimates we get

$$
\left|\mathcal{L}_{2}+\mathcal{L}_{4}\right| \leq \xi(\epsilon)
$$

It is easy to verify that

$$
\left|\mathcal{L}_{3}\right| \leq\left\|\nabla_{x_{h}} \mathbf{v}_{h}\right\|_{L^{\infty}\left(0, \tau ; L^{\infty}(\Omega)\right)} \int_{0}^{\tau} \mathcal{E}_{\epsilon}(t) \mathrm{d} t .
$$

For the term $\mathcal{L}_{5}$, the first observation is that for $x \in \Omega$ we have

$$
P^{\prime \prime}(\tilde{\varrho}(x))-P^{\prime \prime}(1)=(\tilde{\varrho}-1) P^{\prime \prime \prime}(\eta(x)),
$$

where $\eta(x) \in(\min \{1, \tilde{\varrho}\}, \max \{1, \tilde{\varrho}\})$. From the choice of $\tilde{\varrho}=\tilde{\varrho_{\epsilon}}+\epsilon^{m} q_{\epsilon}$ and estimate (4.13), we have

$$
\sup _{x \in \Omega}\left|P^{\prime \prime \prime}(\eta(x))\right| \leq C,
$$

where $C$ is dependent only on $\mathbf{v}_{0}$. 
We rewrite $\mathcal{L}_{5}$ as

$$
\begin{aligned}
\mathcal{L}_{5}= & \frac{1}{\epsilon^{m}} \int_{0}^{\tau} \int_{\Omega}(\tilde{\varrho}-1) P^{\prime \prime \prime}(\eta(x)) \mathbf{m}_{\epsilon} \cdot \nabla_{x} q_{\epsilon} \mathrm{d} x \mathrm{~d} t \\
= & \frac{1}{\epsilon^{m}} \int_{0}^{\tau} \int_{\Omega} \mathbf{m}_{\epsilon} \cdot \nabla_{x} q_{\epsilon}\left(\tilde{\varrho}_{\epsilon}-1\right) P^{\prime \prime \prime}(\eta(x)) \mathrm{d} x \mathrm{~d} t \\
& +\int_{0}^{\tau} \int_{\Omega} q_{\epsilon} \mathbf{m}_{\epsilon} \cdot \nabla_{x} q_{\epsilon} P^{\prime \prime \prime}(\eta(x)) \mathrm{d} x \mathrm{~d} t,
\end{aligned}
$$

By using (4.1), we observe

$$
\begin{aligned}
\left|\mathcal{L}_{5}\right| \leq & \epsilon^{m-2 n}\left\|\mathbf{m}_{\epsilon}\right\|_{L^{\infty}\left(0, T ; L^{2}+L^{4 / 3}\left(\Omega ; \mathbb{R}^{3}\right)\right)}\left\|\nabla_{x} q_{\epsilon}\right\|_{L^{\infty}\left(0, T ; L^{2} \cap L^{4}\left(\Omega ; \mathbb{R}^{3}\right)\right)} \\
& +\left\|\mathbf{m}_{\epsilon}\right\|_{L^{\infty}\left(0, T ; L^{2}+L^{4 / 3}\left(\Omega ; \mathbb{R}^{3}\right)\right)}\left\|q_{\epsilon} \nabla_{x} q_{\epsilon}\right\|_{L^{\infty}\left(0, T ; L^{2} \cap L^{4}\left(\Omega ; \mathbb{R}^{3}\right)\right)},
\end{aligned}
$$

for $\gamma>2$, and

$$
\begin{aligned}
\left|\mathcal{L}_{5}\right| \leq & \epsilon^{\frac{2(m-n)}{\gamma-1}}\left\|\mathbf{m}_{\epsilon}\right\|_{L^{\infty}\left(0, T ; L^{2}+L^{2 \gamma / \gamma+1}\left(\Omega ; \mathbb{R}^{3}\right)\right)}\left\|\nabla_{x} q_{\epsilon}\right\|_{L^{\infty}\left(0, T ; L^{2} \cap L^{(2 \gamma / \gamma+1)^{\prime}}\left(\Omega ; \mathbb{R}^{3}\right)\right)} \\
& +\left\|\mathbf{m}_{\epsilon}\right\|_{L^{\infty}\left(0, T ; L^{2}+L^{2 \gamma / \gamma+1}\left(\Omega ; \mathbb{R}^{3}\right)\right)}\left\|q_{\epsilon} \nabla_{x} q_{\epsilon}\right\|_{L^{\infty}\left(0, T ; L^{2} \cap L^{(2 \gamma / \gamma+1)^{\prime}}\left(\Omega ; \mathbb{R}^{3}\right)\right)},
\end{aligned}
$$

for $1<\gamma \leq 2$, where $\left(\frac{2 \gamma}{\gamma+1}\right)^{\prime}=\frac{2 \gamma}{\gamma-1}$.

In particular, $\frac{m}{2}>n \geq 1$, (4.4) and (4.12) imply

$$
\left|\mathcal{L}_{5}\right| \leq \epsilon^{2(m-n)-1} C, \text { for } \gamma>2,
$$

and

$$
\left|\mathcal{L}_{5}\right| \leq \epsilon^{\frac{(m-2 n)+(m-\gamma+1)}{\gamma-1}} C, \text { for } 1<\gamma<2,
$$

where $C$ is a constant depending on $\mathbf{v}_{0}$ in both cases. Finally we obtain

$$
\mathcal{L}_{5} \leq \xi(\epsilon)
$$

Similarly, we rewrite the term $\mathcal{L}_{6}$ as

$$
\begin{aligned}
\mathcal{L}_{6} & =\frac{1}{\epsilon^{2 m}} \int_{0}^{\tau} \int_{\Omega} \mathbf{m}_{\epsilon} \cdot\left(\tilde{\varrho}-\tilde{\varrho}_{\epsilon}\right) P^{\prime \prime \prime}(\zeta(x)) \nabla_{x} \tilde{\varrho}_{\epsilon} \mathrm{d} x \mathrm{~d} t \\
& =\frac{1}{\epsilon^{m}} \int_{0}^{\tau} \int_{\Omega} \mathbf{m}_{\epsilon} \cdot q_{\epsilon} P^{\prime \prime \prime}(\zeta(x)) \nabla_{x} \tilde{\varrho}_{\epsilon} \mathrm{d} x \mathrm{~d} t,
\end{aligned}
$$

where for each $x, \zeta(x) \in\left(\min \{\tilde{\varrho} \epsilon, \tilde{\varrho}\}, \max \left\{\tilde{\varrho_{\epsilon}}, \tilde{\varrho}\right\}\right)$. Using arguments similar to $\mathcal{L}_{5}$, we have

$$
\begin{aligned}
\left|\mathcal{L}_{6}\right| & \leq \epsilon^{m-2 n}\left\|\mathbf{m}_{\epsilon}\right\|_{L^{\infty}\left(0, T ; L^{2}+L^{2 \gamma / \gamma+1}\left(\Omega ; \mathbb{R}^{3}\right)\right)}\left\|q_{\epsilon}\right\|_{L^{\infty}\left(0, T ; L^{2} \cap L^{(2 \gamma / \gamma+1)^{\prime}}(\Omega)\right)} \\
& \leq \xi(\epsilon) .
\end{aligned}
$$

Now, the choice of $G$ implies

$$
\mathcal{L}_{7}=0
$$


The compatibility of the defect measures (3.5) yields

$$
\left|\mathcal{L}_{8}\right| \leq C \int_{0}^{\tau} \int_{\bar{\Omega}} \mathrm{d} \Re_{e_{\epsilon}} \mathrm{d} t
$$

Therefore, combining all estimates (4.15)-(4.21), we get

$$
\mathcal{E}_{\epsilon}(\tau)+\int_{\bar{\Omega}} \mathrm{d} \Re_{e_{\epsilon}}(\tau, \cdot) \leq \xi(\epsilon)+c \int_{0}^{\tau} \mathcal{E}_{\epsilon}(t) \mathrm{d} t+C \int_{0}^{\tau} \int_{\bar{\Omega}} \mathrm{d} \Re_{e_{\epsilon}} \mathrm{d} t .
$$

We use Grönwall's lemma to infer

$$
\mathcal{E}_{\epsilon}(\tau)+\int_{\bar{\Omega}} \mathrm{d} \Re_{e_{\epsilon}}(\tau, \cdot) \leq \xi(\epsilon) C(T)
$$

where $\xi(\epsilon) \rightarrow 0$ as $\epsilon \rightarrow 0$ and for a.e. $\tau$ with $0 \leq \tau \leq T$. The coercivity of the relative energy functional helps to deduce

$$
\limsup _{\epsilon \rightarrow 0} \int_{K}\left|\frac{\mathbf{m}_{\epsilon}}{\sqrt{\varrho_{\epsilon}}}-\mathbf{v}\right|^{2} \mathrm{~d} x \leq C(T) \limsup _{\epsilon \rightarrow 0} \xi(\epsilon),
$$

where $K \subset \Omega$ is a compact set. Thus, we conclude that $\mathbf{u}=\mathbf{v}_{h}$. Also, we obtain

$$
\frac{\mathbf{m}_{\epsilon}}{\sqrt{\varrho_{\epsilon}}} \rightarrow \mathbf{v} \text { strongly in } L_{\text {loc }}^{1}\left((0, T) \times \Omega ; \mathbb{R}^{3}\right) \text {. }
$$

It ends proof of Theorem 4.2.

\subsection{Proof of Theorem 4.3}

\subsubsection{Uniform bounds and weak convergence}

To obtain a uniform bound, we proceed similarly to Section 4.2.3. First, using $\tilde{\mathbf{u}}=0, \tilde{\varrho}=\hat{\varrho}$ as test functions and well-prepared data(4.3), we obtain the following uniform bounds:

$$
\begin{aligned}
& \text { ess } \sup _{t \in(0, T)}\left\|\frac{\mathbf{m}_{\epsilon}}{\sqrt{\varrho_{\epsilon}}}\right\|_{L^{2}\left(\Omega ; \mathbb{R}^{3}\right)} \leq C, \\
& \text { ess } \sup _{t \in(0, T)}\left\|\left[\frac{\varrho_{\epsilon}-\hat{\varrho}}{\epsilon}\right]_{\mathrm{ess}}\right\|_{L^{2}(\Omega)} \leq C, \\
& \text { ess } \sup _{t \in(0, T)}\left\|\left[\varrho_{\epsilon}\right]_{\mathrm{res}}\right\|_{L^{\gamma}(\Omega)}^{\gamma}+\text { ess } \sup _{t \in(0, T)}\left\|[1]_{\mathrm{res}}\right\|_{L^{\gamma}(\Omega)}^{\gamma} \leq \epsilon^{2} C .
\end{aligned}
$$

This implies that

$$
\begin{aligned}
& \varrho_{\epsilon}^{(1)} \equiv\left(\frac{\varrho_{\epsilon}-\hat{\varrho}}{\epsilon}\right) \rightarrow \varrho^{(1)} \text { weak-(*)ly in } L^{\infty}\left(0, T ; L^{2}+L^{\gamma^{\prime}}(\Omega)\right), \\
& \frac{\mathbf{m}_{\epsilon}}{\sqrt{\varrho_{\epsilon}}} \rightarrow \hat{\mathbf{m}} \text { weak-(*)ly in } L^{\infty}\left(0, T ; L^{2}\left(\Omega ; \mathbb{R}^{3}\right)\right),
\end{aligned}
$$


passing to a suitable subsequence as the case may be, here $\gamma^{\prime}=\min \{2, \gamma\}$.

We also deduce that

$$
\varrho_{\epsilon} \rightarrow \hat{\varrho} \text { in } L^{\infty}\left(0, T ; L^{2}+L^{\gamma^{\prime}}(\Omega)\right) .
$$

Furthermore, we have

$$
\left\|\mathbf{m}_{\epsilon}\right\|_{L^{\infty}\left(0, T ; L^{2}+L^{\grave{\gamma}}\left(\Omega ; \mathbb{R}^{3}\right)\right)} \leq C,
$$

where $\dot{\gamma}=\left\{\frac{4}{3}, \frac{2 \gamma}{\gamma+1}\right\}$. Eventually, for a suitable subsequence, we get

$$
\mathbf{m}_{\epsilon} \rightarrow \mathbf{m} \text { weak-(*)ly in } L^{\infty}\left(0, T ; L^{2}+L^{\grave{\gamma}}\left(\Omega ; \mathbb{R}^{3}\right)\right) .
$$

Letting $\epsilon \rightarrow 0$ in the continuity equation, we infer the incompressibility condition in the weak sense, i.e.,

$$
\int_{0}^{\tau} \int_{\Omega} \mathbf{m} \cdot \nabla_{x} \varphi \mathrm{d} x \mathrm{~d} t=0 .
$$

Define $\mathbf{u}=\frac{\mathbf{m}}{\hat{\varrho}}$. Multiplying momentum equation by $\epsilon$ and letting $\epsilon \rightarrow 0$, we obtain the diagnostic equation

$$
\mathbf{b} \times \mathbf{u}+\nabla_{x}\left(P^{\prime \prime}(\hat{\varrho}) \varrho^{(1)}\right)=0,
$$

in the sense of distributions.

\subsubsection{Strong convergence using relative energy inequality}

Let $(q, \mathbf{v})$ be a strong solution of the above system with initial data $\left(q_{0}, \mathbf{v}_{0}\right)$ satisfying (4.3) with $k \geq 4$. Our goal is to show that $\left(\varrho^{(1)}, \mathbf{u}\right) \equiv(q, \mathbf{v})$. Here we choose appropriate test functions and will show that $\lim _{\epsilon \rightarrow 0} \mathcal{E}_{\epsilon}(t)=0$.

We consider the test functions for the relative energy inequality (3.10) as

$$
\tilde{\mathbf{u}}=\mathbf{v}=\left(\mathbf{v}_{h}, 0\right), \tilde{\varrho}=\hat{\varrho}+\epsilon q .
$$

Thus, we rewrite the relative energy inequality in the following form:

$$
\begin{aligned}
\mathcal{E}_{\epsilon}(\tau) & +\int_{\bar{\Omega}} \mathrm{d} \Re_{e_{\epsilon}}(\tau) \leq \mathcal{E}_{\epsilon}(0) \\
& -\int_{0}^{\tau} \int_{\Omega}\left(\mathbf{m}_{\epsilon}-\varrho_{\epsilon} \mathbf{v}\right) \cdot\left(\partial_{t} \mathbf{v}+\left(\mathbf{v} \cdot \nabla_{x}\right) \mathbf{v}\right) \mathrm{d} x \mathrm{~d} t \\
& -\int_{0}^{\tau} \int_{\Omega}\left(\frac{\left(\mathbf{m}_{\epsilon}-\varrho_{\epsilon} \mathbf{v}\right) \otimes\left(\mathbf{m}_{\epsilon}-\varrho_{\epsilon} \mathbf{v}\right)}{\varrho_{\epsilon}}\right): \nabla_{x} \mathbf{v} \mathrm{d} x \mathrm{~d} t \\
& -\frac{1}{\epsilon^{2}} \int_{0}^{\tau} \int_{\Omega}\left(p\left(\varrho_{\epsilon}\right)-p(\tilde{\varrho})\right) \operatorname{div}_{x} \mathbf{v} \mathrm{d} x \mathrm{~d} t \\
& -\frac{1}{\epsilon} \int_{0}^{\tau} \int_{\Omega} \mathbf{b} \times \mathbf{v} \cdot\left(\mathbf{m}_{\epsilon}-\hat{\varrho} \mathbf{v}\right) \mathrm{d} x \mathrm{~d} t
\end{aligned}
$$




$$
\begin{aligned}
& +\frac{1}{\epsilon^{2}} \int_{0}^{\tau} \int_{\Omega}\left(\hat{\varrho}+\epsilon q-\varrho_{\epsilon}\right) P^{\prime \prime}(\hat{\varrho}+\epsilon q) \partial_{t}(\hat{\varrho}+\epsilon q) \mathrm{d} x \mathrm{~d} t \\
& +\frac{1}{\epsilon^{2}} \int_{0}^{\tau} \int_{\Omega}\left((\hat{\varrho}+\epsilon q) \mathbf{v}-\mathbf{m}_{\epsilon}\right) \cdot \nabla_{x}\left(P^{\prime}(\hat{\varrho}+\epsilon q)-P^{\prime}(\hat{\varrho})\right) \mathrm{d} x \mathrm{~d} t \\
& -\int_{0}^{\tau} \int_{\bar{\Omega}} \nabla_{x} \mathbf{v}: \mathrm{d} \Re_{m_{\epsilon}}(t, \cdot) \mathrm{d} t .
\end{aligned}
$$

Using the fact $\operatorname{div}_{x_{h}} \mathbf{v}=0$ and (3.2), we obtain

$$
\begin{aligned}
\mathcal{E}_{\epsilon}(\tau)+ & \int_{\bar{\Omega}} \mathrm{d} \Re_{e_{\epsilon}}(\tau) \\
\leq & \mathcal{E}_{\epsilon}(0)-\int_{0}^{\tau} \int_{\Omega}\left(\mathbf{m}_{\epsilon}-\varrho_{\epsilon} \mathbf{v}\right) \cdot\left(\partial_{t} \mathbf{v}+\left(\mathbf{v} \cdot \nabla_{x}\right) \mathbf{v}\right) \mathrm{d} x \mathrm{~d} t \\
& +\frac{1}{\epsilon} \int_{0}^{\tau} \int_{\Omega}\left(\hat{\varrho}+\epsilon q-\varrho_{\epsilon}\right) P^{\prime \prime}(\hat{\varrho}+\epsilon q) \partial_{t} q \mathrm{~d} x \mathrm{~d} t \\
& -\int_{0}^{\tau} \int_{\Omega}\left(\frac{\left(\mathbf{m}_{\epsilon}-\varrho_{\epsilon} \mathbf{v}\right) \otimes\left(\mathbf{m}_{\epsilon}-\varrho_{\epsilon} \mathbf{v}\right)}{\varrho_{\epsilon}}\right): \nabla_{x} \mathbf{v} \mathrm{d} x \mathrm{~d} t \\
& -\frac{1}{\epsilon^{2}} \int_{0}^{\tau} \int_{\Omega}\left(\hat{\varrho} \mathbf{v}-\mathbf{m}_{\epsilon}\right) \cdot \nabla_{x}\left(P^{\prime}(\hat{\varrho}+\epsilon q)-P^{\prime}(\hat{\varrho})-\epsilon P^{\prime \prime}(\hat{\varrho}) q\right) \mathrm{d} x \mathrm{~d} t \\
& +\frac{1}{\epsilon} \int_{0}^{\tau} \int_{\Omega} q \mathbf{v} \cdot \nabla_{x}\left(P^{\prime}(\hat{\varrho}+\epsilon q)-P^{\prime}(\hat{\varrho})\right) \mathrm{d} x \mathrm{~d} t \\
& -\int_{0}^{\tau} \int_{\bar{\Omega}} \nabla_{x} \mathbf{v}: \mathrm{d} \Re_{m_{\epsilon}}(t, \cdot) \mathrm{d} t=\Sigma_{i=1}^{7} \mathcal{L}_{i} .
\end{aligned}
$$

Now we want to estimate each term $\mathcal{L}_{i}$ for $i=1, \ldots, 7$. First we notice that, consideration of the well-prepared data (4.3) yields

$$
\mathcal{E}_{\epsilon}\left(\varrho_{0, \epsilon}, \mathbf{m}_{0, \epsilon} \mid \bar{\varrho}+\epsilon q_{0}, \mathbf{v}_{0}\right) \leq\left\|\frac{\mathbf{m}_{0, \epsilon}}{\varrho_{0, \epsilon}}-\mathbf{v}_{0}\right\|_{L^{2}(\Omega)}^{2}+\left\|\varrho_{0, \epsilon}^{(1)}-q_{0}\right\|_{L^{2}(\Omega)}^{2}
$$

This implies

$$
\left|\mathcal{L}_{1}\right| \leq c(\epsilon)
$$

Here $c(\epsilon)$ is a generic function such that $c(\epsilon) \rightarrow 0$ as $\epsilon \rightarrow 0$.

First, we rewrite two terms of $\mathcal{L}_{2}$ and $\mathcal{L}_{3}$ as

$$
\mathbf{m}_{\epsilon}-\varrho_{\epsilon} \mathbf{v}=\left(\mathbf{m}_{\epsilon}-\bar{\varrho} \mathbf{u}\right)+\left(\left(\bar{\varrho}-\varrho_{\epsilon}\right) \mathbf{u}\right)+\left(\varrho_{\epsilon}(\mathbf{u}-\mathbf{v})\right)
$$

and

$$
P^{\prime \prime}(\hat{\varrho}+\epsilon q)=P^{\prime \prime}(\hat{\varrho}+\epsilon q)-P^{\prime \prime}(\hat{\varrho})+P^{\prime \prime}(\hat{\varrho})
$$

Using the weak convergence of the variables, we obtain 


$$
\begin{aligned}
\left|\mathcal{L}_{2}+\mathcal{L}_{3}\right| \leq & c(\epsilon)+\int_{0}^{\tau} \int_{\Omega}(\hat{\varrho} \mathbf{v}-\mathbf{m}) \cdot\left(\partial_{t} \mathbf{v}+\left(\mathbf{v} \cdot \nabla_{x}\right) \mathbf{v}\right) \mathrm{d} x \mathrm{~d} t \\
& +\int_{0}^{\tau} \int_{\Omega}\left(q-\varrho^{(1)}\right) \partial_{t}\left(P^{\prime \prime}(\hat{\varrho}) q\right) \mathrm{d} x \mathrm{~d} t .
\end{aligned}
$$

We claim that

$$
\begin{aligned}
\int_{0}^{\tau} & \int_{\Omega}(\hat{\varrho} \mathbf{v}-\mathbf{m}) \cdot\left(\partial_{t} \mathbf{v}+\left(\mathbf{v} \cdot \nabla_{x}\right) \mathbf{v}\right) \mathrm{d} x \mathrm{~d} t \\
& +\int_{0}^{\tau} \int_{\Omega}\left(q-\varrho^{(1)}\right) \partial_{t}\left(P^{\prime \prime}(\hat{\varrho}) q\right) \mathrm{d} x \mathrm{~d} t=0 .
\end{aligned}
$$

To prove the above claim, first we observe that

$$
\mathbf{v} \cdot \partial_{t}(\hat{\varrho} \mathbf{v})+q \partial_{t}\left(P^{\prime}(\hat{\varrho}) q\right)=\frac{1}{2} \partial_{t}\left(\hat{\varrho}|\mathbf{v}|^{2}+P^{\prime}(\hat{\varrho}) q^{2}\right) .
$$

Since $(q, \mathbf{v})$ solves $(2.4)$, multiplying (3.3) by $q$ we get

$$
\int_{0}^{\tau} \int_{\Omega} \mathbf{v} \cdot \partial_{t}(\hat{\varrho} \mathbf{v})+q \partial_{t}\left(P^{\prime}(\hat{\varrho}) q\right) \mathrm{d} x \mathrm{~d} t=0 .
$$

Now we use (4.22) and (3.2) to deduce

$$
\int_{0}^{\tau} \int_{\Omega}\left(\mathbf{m} \cdot \partial_{t} \mathbf{v}+\varrho^{(1)} \partial_{t}\left(P^{\prime \prime}(\hat{\varrho}) q\right)\right) \mathrm{d} x \mathrm{~d} t=\int_{0}^{\tau} \int_{\Omega} \mathbf{b} \times \mathbf{m} \cdot\left(P^{\prime \prime}(\hat{\varrho}) \mathbf{v}\right) \mathrm{d} x \mathrm{~d} t
$$

and

$$
\begin{aligned}
\int_{0}^{\tau} \int_{\Omega} \mathbf{b} \times \mathbf{m} \cdot\left(P^{\prime \prime}(\hat{\varrho}) \mathbf{v}\right) \mathrm{d} x \mathrm{~d} t+\int_{0}^{\tau} \int_{\Omega} \mathbf{m} \cdot\left(\mathbf{v} \cdot \nabla_{x}\right) \mathbf{v} \mathrm{d} x \mathrm{~d} t \\
\quad=\int_{0}^{\tau} \mathbf{m} \cdot \nabla_{x_{h}}\left(\frac{1}{2}|\mathbf{v}|^{2}\right) \mathrm{d} x \mathrm{~d} t=0 .
\end{aligned}
$$

Hence, we achieve (4.24) and it implies

$$
\left|\mathcal{L}_{2}+\mathcal{L}_{3}\right| \leq c(\epsilon)
$$

From the definition of relative energy, we obtain

$$
\left|\mathcal{L}_{4}\right| \leq \int_{0}^{\tau} \mathcal{E}_{\epsilon}(t) \mathrm{d} t
$$

Since $0<\hat{\varrho} \in C^{3}([0,1])$, we verify that

$$
\frac{1}{\epsilon} \nabla_{x}\left(P^{\prime}(\hat{\varrho}+\epsilon q)-P^{\prime}(\hat{\varrho})\right) \rightarrow \nabla_{x}\left(P^{\prime \prime}(\hat{\varrho}) q\right) \text { in } L^{\infty}\left(0, T ; L^{\infty} \cap L^{2}(\Omega)\right)
$$


and

$$
\begin{aligned}
\frac{1}{\epsilon^{2}} \nabla_{x}\left(P^{\prime}(\hat{\varrho}+\epsilon q)\right. & \left.-P^{\prime}(\hat{\varrho})-\epsilon P^{\prime \prime}(\hat{\varrho}) q\right) \\
& \rightarrow \nabla_{x}\left(\frac{1}{2} P^{\prime \prime \prime}(\hat{\varrho})\right) \text { in } L^{\infty}\left(0, T ; L^{\infty} \cap L^{2}(\Omega)\right)
\end{aligned}
$$

The above relation implies

$$
\lim _{\epsilon \rightarrow 0} \mathcal{L}_{5}=\int_{0}^{\tau} \int_{\Omega}(\hat{\varrho} \mathbf{v}-\mathbf{m}) \cdot \nabla_{x}\left(\frac{1}{2} P^{\prime \prime \prime}(\hat{\varrho})\right) \mathrm{d} x \mathrm{~d} t=0
$$

and

$$
\lim _{\epsilon \rightarrow 0} \mathcal{L}_{6}=\int_{0}^{\tau} \int_{\Omega} q \mathbf{v} \rightarrow \nabla_{x}\left(P^{\prime \prime}(\hat{\varrho}) q\right) \mathrm{d} x \mathrm{~d} t=0
$$

We obtain

$$
\left|\mathcal{L}_{7}\right| \leq C \int_{0}^{\tau} \int_{\bar{\Omega}} \mathbb{I}: \mathrm{d} \mathfrak{C}_{m_{\epsilon}}(t, \cdot) \mathrm{d} t
$$

Combining (4.23)-(4.29), we have that

$$
\mathcal{E}_{\epsilon}(\tau)+\int_{\bar{\Omega}} \mathrm{d} \mathfrak{C}_{e_{\epsilon}} \leq c(\epsilon)+\int_{0}^{\tau} \mathcal{E}_{\epsilon}(t) \mathrm{d} t+C \int_{0}^{\tau} \int_{\bar{\Omega}} \mathrm{d} \operatorname{Trace}\left(\mathfrak{C}_{m_{\epsilon}}\right) \mathrm{d} t
$$

for a.e. $\tau \in(0, T)$. Finally, using the compatibility of turbulent defect measures and Grönwall's lemma, we infer

$$
\mathcal{E}_{\epsilon}(\tau)+\int_{\bar{\Omega}} \mathrm{d} \mathfrak{C}_{e_{\epsilon}} \leq c(\epsilon) \leq c(\epsilon) C(T),
$$

where $c(\epsilon) \rightarrow 0$ as $\epsilon \rightarrow 0$. Therefore, we obtain our desired result

$$
\lim _{\epsilon \rightarrow 0} \mathcal{E}_{\epsilon}(\tau)=0
$$

Now using coercivity of the relative energy functional as stated in Remark 3.5, we say

$$
\varrho^{(1)}=q \text { and } \mathbf{v}=\mathbf{u}
$$

Moreover, we use coercivity together with (4.30) to conclude

$$
\begin{aligned}
& \frac{\varrho_{\epsilon}-\hat{\varrho}}{\epsilon} \rightarrow q \text { strongly in } L_{\text {loc }}^{1}((0, T) \times \Omega), \\
& \frac{\mathbf{m}_{\epsilon}}{\sqrt{\varrho_{\epsilon}}} \rightarrow \sqrt{\hat{\varrho}} \mathbf{v} \text { strongly in } L_{\text {loc }}^{1}\left((0, T) \times \Omega ; \mathbb{R}^{3}\right) .
\end{aligned}
$$

This completes the proof of Theorem 4.3. 


\section{Extension to the Navier-Stokes system}

Here our goal is to give a proper definition of a dissipative solution for NavierStokes equation. We consider another characteristic number, i.e., Reynolds number. Additional assumption with high Reynolds number limit along with other characteristic number limits helps us to obtain the same target system.

5.1. Definition of dissipative solution for Navier-Stokes system

Let $\varrho$ be the density and $\mathbf{u}$ be the velocity. In time-space cylinder $Q_{T}=(0, T) \times \Omega$, we consider:

- Conservation of mass

$$
\partial_{t} \varrho+\operatorname{div}_{x}(\varrho \mathbf{u})=0 .
$$

- Conservation of momentum

$$
\begin{aligned}
& \partial_{t}(\varrho \mathbf{u})+\operatorname{div}_{x}(\varrho \mathbf{u} \otimes \mathbf{u})+\frac{1}{\mathrm{Ma}^{2}} \nabla_{x} p(\varrho)+\frac{1}{\mathrm{Ro}} \mathbf{b} \times \varrho \mathbf{u} \\
& =\frac{1}{\mathrm{Re}} \operatorname{div}_{x} \mathbb{S}\left(\nabla_{x} \mathbf{u}\right)+\frac{1}{\mathrm{Fr}^{2}} \varrho \nabla_{x} G .
\end{aligned}
$$

- Constitutive relation Here $\mathbb{S}\left(\nabla_{x} \mathbf{u}\right)$ is the Newtonian stress tensor defined by

$$
\mathbb{S}\left(\nabla_{x} \mathbf{u}\right)=\mu\left(\frac{\nabla_{x} \mathbf{u}+\nabla_{x}^{T} \mathbf{u}}{2}-\frac{1}{d}\left(\operatorname{div}_{x} \mathbf{u}\right) \mathbb{I}\right)+\lambda\left(\operatorname{div}_{x} \mathbf{u}\right) \mathbb{I},
$$

where $\mu>0$ and $\lambda>0$ are the shear and bulk viscosity coefficients, respectively.

- The scaled system contains all specified characteristic numbers as in (1.5) along with,

- Re- Reynolds number.

Here we consider,

$$
\mathrm{Ma} \approx \epsilon^{m}, \text { Ro } \approx \epsilon, \operatorname{Re} \approx \epsilon^{-\alpha}, \text { Fr } \approx \epsilon^{n} \text { for } \epsilon>0, m, n, \alpha>0 \text { and } \frac{m}{2}>n \geq 1
$$

- Pressure law In an isentropic setting, the pressure $p$ and the density $\varrho$ of the fluid are interrelated by

$$
p(\varrho)=a \varrho^{\gamma}, a>0, \gamma>1 .
$$

- Boundary condition Here we consider a complete slip condition for the velocity on the horizontal boundary, i.e.,

$$
\left.\mathbf{u} \cdot \mathbf{n}\right|_{\partial \Omega}=\left.\left[\mathbb{S}\left(\nabla_{x} \mathbf{u}\right) \cdot \mathbf{n}\right]_{\tan }\right|_{\partial \Omega}=0, \mathbf{n}=(0,0, \pm 1) .
$$


- Far field condition Let $\left(\tilde{\varrho}_{\epsilon}, \mathbf{0}\right)$ be a static solution, we assume the condition as

$$
\left|\varrho-\tilde{\varrho}_{\epsilon}\right| \rightarrow 0, \mathbf{u} \rightarrow \mathbf{0} \text { as }\left|x_{h}\right| \rightarrow \infty
$$

- Initial data For each $\epsilon>0$, we supplement the initial data as

$$
\varrho(0, \cdot)=\varrho_{\epsilon, 0},(\varrho \mathbf{u})(0, \cdot)=(\varrho \mathbf{u})_{\epsilon, 0} .
$$

Here we provide the definition of dissipative solution for the Navier-Stokes system.

Definition 5.1. Let $\epsilon>0$ and $\tilde{\varrho}_{\epsilon}>0$. We say a pair of functions $\left(\varrho_{\epsilon}, \mathbf{u}_{\epsilon}\right)$ with

$$
\begin{aligned}
& \varrho_{\epsilon}-\tilde{\varrho_{\epsilon}} \in C_{\text {weak }}\left([0, T] ; L^{2}+L^{\gamma}(\Omega)\right), \varrho_{\epsilon} \geq 0, \\
& \varrho_{\epsilon} \mathbf{u}_{\epsilon} \in C_{\text {weak }}\left([0, T] ; L^{2}+L^{\frac{2 \gamma}{\gamma+1}}(\Omega)\right), \\
& \mathbf{u}_{\epsilon} \in L^{2}\left(0, T ; W^{1,2}(\Omega)\right),\left.\mathbf{u}_{\epsilon} \cdot \mathbf{n}\right|_{\partial \Omega}=0
\end{aligned}
$$

is a dissipative solution to (5.1)-(5.7) with initial data $\left(\varrho_{0, \epsilon},(\varrho \mathbf{u})_{0, \epsilon}\right)$ satisfying

$$
\begin{aligned}
\varrho_{0, \epsilon} & \geq 0, \quad E_{0, \epsilon}=\int_{\Omega}\left(\frac{1}{2} \frac{\left|(\varrho \mathbf{u})_{0, \epsilon}\right|^{2}}{\varrho_{0, \epsilon}}+P\left(\varrho_{0, \epsilon}\right)-\left(\varrho_{0, \epsilon}-\tilde{\varrho}_{\epsilon}\right) P^{\prime}\left(\tilde{\varrho}_{\epsilon}\right)-P\left(\tilde{\varrho}_{\epsilon}\right)\right) \mathrm{d} x \\
& <\infty,
\end{aligned}
$$

if there exist the turbulent defect measures

$$
\Re_{m_{\epsilon}} \in L^{\infty}\left(0, T ; \mathcal{M}^{+}\left(\bar{\Omega} ; \mathbb{R}_{\mathrm{sym}}^{d \times d}\right)\right), \mathfrak{R}_{e_{\epsilon}} \in L^{\infty}\left(0, T ; \mathcal{M}^{+}(\bar{\Omega})\right),
$$

satisfying compatibility condition

$$
\lambda_{1} \text { Trace } \Re_{m_{\epsilon}} \leq \mathfrak{R}_{e_{\epsilon}} \leq \lambda_{2} \text { Trace } \Re_{m_{\epsilon}}, \lambda_{1}, \lambda_{2}>0,
$$

such that the following holds:

- Equation of continuity For any $\tau \in(0, T)$ and any $\varphi \in C_{c}^{1}([0, T] \times \bar{\Omega})$ it holds

$$
\left[\int_{\Omega} \varrho_{\epsilon} \varphi \mathrm{d} x\right]_{t=0}^{t=\tau}=\int_{0}^{\tau} \int_{\Omega}\left[\varrho_{\epsilon} \partial_{t} \varphi+\varrho_{\epsilon} \mathbf{u}_{\epsilon} \cdot \nabla_{x} \varphi\right] \mathrm{d} x \mathrm{~d} t ;
$$

- Momentum equation For any $\tau \in(0, T)$ and any $\varphi \in C_{c}^{1}\left([0, T] \times \Omega\right.$; $\left.\mathbb{R}^{d}\right)$ with $\left.\boldsymbol{\varphi} \cdot \mathbf{n}\right|_{\partial \Omega}=0$, it holds

$$
\begin{aligned}
& {\left[\int_{\Omega} \varrho_{\epsilon} \mathbf{u}_{\epsilon}(\tau, \cdot) \cdot \boldsymbol{\varphi}(\tau, \cdot) \mathrm{d} x\right]_{t=0}^{t=\tau}} \\
& =\int_{0}^{\tau} \int_{\Omega}\left[\varrho_{\epsilon} \mathbf{u}_{\epsilon} \cdot \partial_{t} \boldsymbol{\varphi}+\varrho_{\epsilon} \mathbf{u}_{\epsilon} \otimes \mathbf{u}_{\epsilon}: \nabla_{x} \boldsymbol{\varphi}+\frac{1}{\epsilon^{2 m}} p\left(\varrho_{\epsilon}\right) \operatorname{div}_{x} \boldsymbol{\varphi}+\frac{1}{\epsilon} \mathbf{b} \times \varrho_{\epsilon} \mathbf{u}_{\epsilon} \cdot \boldsymbol{\varphi}\right] \mathrm{d} x \mathrm{~d} t \\
& \quad-\int_{0}^{\tau} \int_{\Omega}\left[\epsilon^{\alpha} \mathbb{S}\left(\nabla_{x} \mathbf{u}\right): \nabla_{x} \boldsymbol{\varphi}-\frac{1}{\epsilon^{2 n}} \varrho_{\epsilon} \nabla_{x} G \cdot \boldsymbol{\varphi}\right] \mathrm{d} x \mathrm{~d} t+\int_{0}^{\tau} \int_{\bar{\Omega}} \nabla_{x} \boldsymbol{\varphi}: \mathrm{d} \Re_{m_{\epsilon}} \mathrm{d} t
\end{aligned}
$$


- Energy inequality The total energy $E$ is defined in $[0, T)$ as

$$
E_{\epsilon}(\tau)=\int_{\Omega}\left(\frac{1}{2} \varrho_{\epsilon}\left|\mathbf{u}_{\epsilon}\right|^{2}+\frac{1}{\epsilon^{2 m}}\left(P\left(\varrho_{\epsilon}\right)-\left(\varrho_{\epsilon}-\tilde{\varrho}_{\epsilon}\right) P^{\prime}\left(\tilde{\varrho}_{\epsilon}\right)\right)-P\left(\tilde{\varrho}_{\epsilon}\right)\right)(\tau, \cdot) \mathrm{d} x ;
$$

It satisfies

$$
E_{\epsilon}(\tau)+\epsilon^{\alpha} \int_{0}^{\tau} \int_{\Omega} \mathbb{S}\left(\nabla_{x} \mathbf{u}\right): \nabla_{x} \mathbf{u} \mathrm{d} x \mathrm{~d} t+\int_{\bar{\Omega}} \mathrm{d} \Re_{e_{\epsilon}}(\tau, \cdot) \leq E_{0, \epsilon}
$$

for a.a. $\tau>0$.

Remark 5.2. The class of test functions in the momentum equation corresponds to the complete slip (or Navier slip) boundary conditions. These are necessary to avoid problems with boundary layer.

Theorem 5.3. Suppose $\Omega$ be the domain specified above and the pressure follows (5.5). If $\left(\varrho_{0, \epsilon},(\varrho \mathbf{u})_{0, \epsilon}\right)$ satisfies (5.8), then there exists a dissipative solution as defined above.

We prove the existence theorem for $\epsilon=1$.

Proof. Here we give an extended outline of the proof. We know that the existence theory in the class of finite energy weak solutions was developed by Lions [30] and later extended by Feireisl [16] to the so far subcritical exponent $\gamma>\frac{3}{2}$. For an unbounded domain, similar result has been proposed by Novotný and Pokorný in [32].

Here our goal is to add $\delta \nabla_{x} \varrho^{\Gamma}$ in the momentum equation with $\Gamma \geq \frac{3}{2}$ so that we use the existing results to obtain a finite energy weak solution for the system and we denote it by $\left(\varrho_{\delta}, \mathbf{u}_{\delta}\right)$. Then we will show that this approximate solution converges to a dissipative solution of above described system.

This motivates the following approximate problem:

$$
\begin{aligned}
& \partial_{t} \varrho_{\delta}+\operatorname{div}_{x}\left(\varrho_{\delta} \mathbf{u}_{\delta}\right)=0, \\
& \partial_{t}\left(\varrho_{\delta} \mathbf{u}_{\delta}\right)+\operatorname{div}_{x}\left(\varrho_{\delta} \mathbf{u}_{\delta} \otimes \mathbf{u}_{\delta}\right)+\nabla_{x} p\left(\varrho_{\delta}\right)+\delta \nabla_{x} \varrho_{\delta}^{\Gamma}+\mathbf{b} \times \varrho_{\delta} \mathbf{u}_{\delta} \\
& \quad=\operatorname{div}_{x} \mathbb{S}\left(\nabla_{x} \mathbf{u}_{\delta}\right)+\varrho_{\delta} \nabla_{x} G, \\
& \left.\mathbf{u}_{\delta} \cdot \mathbf{n}\right|_{\partial \Omega}=\left.\left[\mathbb{S}\left(\nabla_{x} \mathbf{u}_{\delta}\right) \cdot \mathbf{n}\right]_{\tan }\right|_{\partial \Omega}=0,
\end{aligned}
$$

with $\Gamma>\frac{3}{2}$. We assume that for each $\delta>0$ a static solution of the approximate problem $\tilde{\varrho}_{\delta}$ has the following property

$$
\sup _{x_{3} \in[0,1]}\left|\tilde{\varrho}_{\delta}-\tilde{\varrho}\right| \leq \delta,
$$

where $\tilde{\varrho}>0$ is a static solution for the Navier-Stokes problem with $\nabla_{x} P^{\prime}(\tilde{\varrho})=$ $\nabla_{x} G$. Further we assume that for the above-mentioned problem, initial condition 
$\left\{\varrho_{\delta, 0},(\varrho \mathbf{u})_{\delta, 0}\right\}$ belongs to a certain regularity class for which weak solution exists. As an additional assumption, we have

$$
\begin{gathered}
E_{0, \delta}^{\delta}=\int_{\Omega}\left(\frac{1}{2} \frac{\mid\left(\varrho_{\mathbf{u})\left._{0, \delta}\right|^{2}}\right.}{\varrho_{0, \delta}}+H\left(\varrho_{0, \delta}\right)-\left(\varrho_{0, \delta}-\tilde{\varrho}_{\delta}\right) H^{\prime}\left(\tilde{\varrho}_{\delta}\right)-H\left(\tilde{\varrho}_{\delta}\right)\right) \mathrm{d} x \\
\rightarrow E_{0}=\int_{\Omega}\left(\frac{1}{2} \frac{\left|\left(\varrho_{\mathbf{u}}\right)_{0}\right|^{2}}{\varrho_{0}}+P\left(\varrho_{0}\right)-\left(\varrho_{0}-\tilde{\varrho}\right) P^{\prime}(\tilde{\varrho})-P(\tilde{\varrho})\right) \mathrm{d} x,
\end{gathered}
$$

where $H(s)=\frac{1}{\gamma-1} p(s)+\delta \frac{1}{\Gamma-1} s^{\Gamma}$.

Clearly from existence of weak solution, we have several apriori bounds, i.e.,

$$
\begin{aligned}
& \left\|\varrho_{\delta}-\tilde{\varrho}_{\delta}\right\|_{L^{\infty}\left(0, T ; L^{2}+L^{\gamma}(\Omega)\right)} \leq C, \\
& \left\|\sqrt{\varrho_{\delta}} \mathbf{u}_{\delta}\right\|_{L^{2}\left(\Omega ; \mathbb{R}^{3}\right)} \leq C, \\
& \left\|\mathbf{u}_{\delta}\right\|_{L^{2}\left(0, T ; W^{1,2}\left(\Omega: \mathbb{R}^{3}\right)\right.} \leq C, \\
& \left\|H\left(\varrho_{\delta}\right)-\left(\varrho_{\delta}-\tilde{\varrho}_{\delta}\right) H^{\prime}\left(\tilde{\varrho}_{\delta}\right)-H\left(\tilde{\varrho}_{\delta}\right)\right\|_{L^{\infty}\left(0, T ; L^{1}(\Omega)\right)} \leq C .
\end{aligned}
$$

As a consequence of that, we obtain

$$
\begin{aligned}
& \left\|\varrho_{\delta} \mathbf{u}_{\delta}\right\|_{L^{\infty}\left(0, T ; L^{2}+L^{2 \gamma / \gamma+1}\left(\Omega ; \mathbb{R}^{3}\right)\right)} \leq C, \\
& \text { ess } \sup _{t \in[0, T]} \int_{\Omega} \delta \varrho_{\delta}^{\Gamma} \mathrm{d} x \leq C .
\end{aligned}
$$

From the above bounds, we get

$$
\begin{aligned}
& \varrho_{\delta}-\tilde{\varrho}_{\delta} \rightarrow \varrho-\tilde{\varrho} \text { weak-(*)ly in } L^{\infty}\left(0, T ; L^{2}+L^{\gamma}(\Omega)\right), \\
& \mathbf{u}_{\delta} \rightarrow \mathbf{u} \text { weakly in } L^{2}\left(0, T ; W^{1,2}\left(\Omega: \mathbb{R}^{3}\right) .\right.
\end{aligned}
$$

We use Abbatiello et al. [1, Lemma 8.1 (Appendix)] to conclude

$$
\varrho_{\delta} \mathbf{u}_{\delta} \rightarrow \varrho \mathbf{u} \text { weak-(*)ly in } L^{\infty}\left(0, T ; L^{2}+L^{2 \gamma / \gamma+1}(\Omega)\right) .
$$

Let us introduce the conservative variable $\mathbf{m}_{\delta}=\varrho_{\delta} \mathbf{u}_{\delta}$. In terms of momentum, we rewrite kinetic energy as

$$
\left(\varrho_{\delta}, \mathbf{m}_{\delta}\right) \mapsto \frac{1}{2} \frac{\left|\mathbf{m}_{\delta}\right|^{2}}{\varrho_{\delta}}= \begin{cases}\frac{1}{2} \frac{\left|\left(\varrho_{\delta} \mathbf{u}_{\delta}\right)\right|^{2}}{\varrho_{\delta}} & \text { if }, \varrho_{\delta} \neq 0, \mathbf{m}_{\delta} \neq 0, \\ 0 & \text { if } \varrho_{\delta}=0, \mathbf{m}_{\delta}=0 \\ \infty & \text { if } \varrho_{\delta}=0, \mathbf{m}_{\delta} \neq 0 .\end{cases}
$$

As an observation, we have the above map as convex 1.s.c. From energy inequality, it is worth to notice that it is $\infty$ only on a measure zero set in $(0, T) \times \Omega$. Using convexity of $p(\cdot)$ and $[\varrho, \mathbf{m}] \mapsto \frac{\mathbf{m} \times \mathbf{m}}{\varrho}$, and also using fact that $L^{1}(\Omega)$ continuously embedded in $\mathcal{M}(\bar{\Omega})$, we conclude

$$
\begin{aligned}
& \frac{\mathbf{m}_{\delta} \times \mathbf{m}_{\delta}}{\varrho_{\delta}} \rightarrow \frac{\overline{\mathbf{m} \times \mathbf{m}}}{\varrho} \text { weak-(*)ly in } L^{\infty}\left(0, T ; \mathcal{M}\left(\bar{\Omega} ; \mathbb{R}_{\mathrm{sym}}^{d \times d}\right)\right), \\
& p\left(\varrho_{\delta}\right) \rightarrow \overline{p(\varrho)} \text { weak-(*)ly in } L^{\infty}(0, T ; \mathcal{M}(\bar{\Omega})), \\
& \delta \varrho_{\delta}^{\Gamma}(t, \cdot) \rightarrow \zeta \text { weak-(*)ly in } L^{\infty}(0, T ; \mathcal{M}(\bar{\Omega})) .
\end{aligned}
$$


We choose

$$
\Re_{m}=\left[\frac{\overline{\mathbf{m} \times \mathbf{m}}}{\varrho}-\frac{\mathbf{m} \times \mathbf{m}}{\varrho}\right]+[\overline{p(\varrho)}-p(\varrho)+\zeta] \mathbb{I},
$$

and

$$
\mathfrak{R}_{e}=\overline{\frac{1}{2} \frac{|\mathbf{m}|^{2}}{\varrho}}-\frac{1}{2} \frac{|\mathbf{m}|^{2}}{\varrho}+\overline{P(\varrho)}-P(\varrho)+\frac{1}{\Gamma-1} \zeta .
$$

The compatibility of two turbulent defect measures is clear from above equations. Arguing similarly as in Breit et al. [7], we obtain

$$
\Re_{m} \in L^{\infty}\left(0, T ; \mathcal{M}^{+}\left(\bar{\Omega} ; \mathbb{R}_{\mathrm{sym}}^{d \times d}\right)\right), \Re_{e} \in L^{\infty}\left(0, T ; \mathcal{M}^{+}(\bar{\Omega})\right) .
$$

Now we are in a position to conclude that $\varrho, \mathbf{u}, \Re_{m}$ and $\mathfrak{R}_{e}$ is a dissipative solution for the Navier-Stokes equation.

Finally, we state the theorem:

Theorem 5.4. Let pressure p follows (5.5). We assume that the initial data are wellprepared. We also consider $\mathbf{v}_{0} \in W^{k, 2}\left(\mathbb{R}^{2}\right)$ with $k \geq 3$. Let $\left(\varrho_{\epsilon}, \mathbf{u}_{\epsilon}\right)$ be a dissipative solution as in Definition 5.1 in $(0, T) \times \Omega$. Then,

$$
\begin{aligned}
& \text { ess } \sup _{t \in(0, T)}\left\|\varrho_{\epsilon}-\tilde{\varrho}_{\epsilon}\right\|_{\left(L^{2}+L^{\gamma}\right)(\Omega)} \leq \epsilon^{m} c, \\
& \sqrt{\varrho_{\epsilon}} \mathbf{u}_{\epsilon} \rightarrow \mathbf{v}\left\{\begin{array}{l}
\text { weak- }(*) \text { ly in } L^{\infty}\left(0, T ; L^{2}\left(\Omega ; \mathbb{R}^{3}\right)\right), \\
\text { strongly in } L_{\text {loc }}^{1}\left((0, T) \times \Omega ; \mathbb{R}^{3}\right),
\end{array}\right.
\end{aligned}
$$

where $\mathbf{v}=\left(\mathbf{v}_{h}, 0\right)$ and $\mathbf{v}_{h}$ is the unique solution of Euler system (2.2) with initial data $\mathbf{v}_{0}$.

Proof. The proof is similar as before only we have to consider few extra terms, see [24].

\section{Acknowledgements}

The work of N. Chaudhuri was partly supported by EPSRC Early Career Fellowship no. EP /V000586/1. The author would like to thank Prof. E. Feireisl for his valuable comments. The author also thanks the unknown reviewer(s) for necessary suggestions to improve the manuscript.

Open Access. This article is licensed under a Creative Commons Attribution 4.0 International License, which permits use, sharing, adaptation, distribution and reproduction in any medium or format, as long as you give appropriate credit to the original author(s) and the source, provide a link to the Creative Commons licence, and indicate if changes were made. The images or other third party material in this article are included in the article's Creative Commons licence, unless indicated otherwise in a credit line to the material. If material is not included in the article's Creative Commons licence and your intended use 
is not permitted by statutory regulation or exceeds the permitted use, you will need to obtain permission directly from the copyright holder. To view a copy of this licence, visit http://creativecommons.org/licenses/ by $/ 4.0 /$.

Publisher's Note Springer Nature remains neutral with regard to jurisdictional claims in published maps and institutional affiliations.

\section{REFERENCES}

[1] A. Abbatiello, E. Feireisl, and A. Novotny, Generalized solutions to models of compressible viscous fluids (2019).

[2] J. J. Alibert and G. Bouchitté, Non-uniform integrability and generalized Young measures, J. Convex Anal. 4 (1997), no. 1, 129-147.

[3] A. Babin, A. Mahalov, and B. Nicolaenko, Global regularity of 3D rotating Navier-Stokes equations for resonant domains, Indiana Univ. Math. J. 48 (1999), no. 3, 1133-1176.

[4] A. Babin, A. Mahalov, and B. Nicolaenko, 3D Navier-Stokes and Euler equations with initial data characterized by uniformly large vorticity, Indiana Univ. Math. J. 50 (2001), no. Special Issue, 1-35. Dedicated to Professors Ciprian Foias and Roger Temam (Bloomington, IN, 2000).

[5] D. Basarić, Vanishing viscosity limit for the compressible Navier-Stokes system via measure-valued solutions, arXiv e-prints (2019Mar), arXiv:1903.05886.

[6] D. Breit, E. Feireisl, and M. Hofmanová, Dissipative solutions and semiflow selection for the complete euler system, arXiv e-prints (2019 Apr), arXiv:1904.00622.

[7] D. Breit, E. Feireisl, and M. Hofmanová, Generalized solutions to models of inviscid fluids, arXiv e-prints (2019 Jul), arXiv:1907.00757.

[8] J. Březina and E. Feireisl, Measure-valued solutions to the complete Euler system, J. Math. Soc. Japan 70 (2018), no. 4, 1227-1245.

[9] G. Bruell and E. Feireisl, On a singular limit for stratified compressible fluids, arXiv e-prints (2018 Feb), arXiv:1802.10340.

[10] J. Březina, Existence of measure-valued solutions to a complete Euler system for a perfect gas, arXiv e-prints (2018 May), arXiv:1805.05570.

[11] J. Březina and V. Mácha, Low stratifucation of the complete Euler system, arXiv e-prints (2018 Dec), arXiv:1812.08465.

[12] J. Březina and E. Feireisl, Measure-valued solutions to the complete Euler system revisited, Z. Angew. Math. Phys. 69 (2018), no. 3, Paper No. 57, 17.

[13] J. Y. Chemin, B. Desjardins, I. Gallagher, and E. Grenier, Mathematical geophysics, Oxford Lecture Series in Mathematics and its Applications, vol. 32, The Clarendon Press, Oxford University Press, Oxford, 2006. An introduction to rotating fluids and the Navier-Stokes equations.

[14] D. G. Ebin, The motion of slightly compressible fluids viewed as a motion with strong constraining force, Ann. Math. (2) 105 (1977), no. 1, 141-200.

[15] D. G. Ebin, Viscous fluids in a domain with frictionless boundary, Global analysis-analysis on manifolds, 1983, pp. 93-110.

[16] E. Feireisl, Dynamics of viscous compressible fluids, Oxford Lecture Series in Mathematics and its Applications, vol. 26, Oxford University Press, Oxford, 2004.

[17] E. Feireisl, I. Gallagher, D. Gerard-Varet, and A. Novotný, Multi-scale analysis of compressible viscous and rotating fluids, Comm. Math. Phys. 314 (2012), no. 3, 641-670.

[18] E. Feireisl, I. Gallagher, and A. Novotný, A singular limit for compressible rotating fluids, SIAM J. Math. Anal. 44 (2012), no. 1, 192-205.

[19] E. Feireisl, P. Gwiazda, A. Świerczewska-Gwiazda, and E. Wiedemann, Dissipative measure-valued solutions to the compressible Navier-Stokes system, Calc. Var. Partial Differential Equations 55 (2016), no. 6, Art. 141, 20.

[20] E. Feireisl, C. Klingenberg, and S. Markfelder, On the low mach number limit for the compressible Euler system, SIAM J. Math. Anal. 51 (2019), no. 2, 1496-1513. 
[21] E. Feireisl and M. Lukáčová-Medvidová, Convergence of a mixed finite element-finite volume scheme for the isentropic Navier-Stokes system via dissipative measure-valued solutions, Found. Comput. Math. 18 (2018), no. 3, 703-730.

[22] E Feireisl, M Lukacova-Medvidova, and H Mizerova, $\mathcal{K}$-convergence as a new tool in numerical analysis, arXiv e-prints (2019 Mar), arXiv:1904.00297.

[23] E. Feireisl and A. Novotný, Singular limits in thermodynamics of viscous fluids, Advances in Mathematical Fluid Mechanics, Birkhäuser Verlag, Basel (2009).

[24] E. Feireisl and A. Novotný, Multiple scales and singular limits for compressible rotating fluids with general initial data, Comm. Partial Differential Equations 39 (2014), no. 6, 1104-1127.

[25] E. Feireisl and A. Novotný, Scale interactions in compressible rotating fluids, Ann. Mat. Pura Appl. (4) 193 (2014), no. 6, 1703-1725.

[26] P. Gwiazda, A. Świerczewska-Gwiazda, and E. Wiedemann, Weak-strong uniqueness for measurevalued solutions of some compressible fluid models, Nonlinearity 28 (2015), no. 11, 3873-3890.

[27] T. Kato and C. Y. Lai, Nonlinear evolution equations and the Euler flow, J. Funct. Anal. 56 (1984), no. $1,15-28$.

[28] S. Klainerman and A. Majda, Singular limits of quasilinear hyperbolic systems with large parameters and the incompressible limit of compressible fluids, Comm. Pure Appl. Math. 34 (1981), no. 4, 481-524.

[29] Y. Li, Singular limit for rotating compressible fluids with centrifugal force in a finite cylinder (2019).

[30] P. L. Lions, Mathematical topics in fluid mechanics. Vol. 2, Oxford Lecture Series in Mathematics and its Applications, vol. 10, The Clarendon Press, Oxford University Press, New York, 1998. Compressible models, Oxford Science Publications.

[31] Š. Nečasová and T. Tang, On a singular limit for the compressible rotating Euler system, J. Math. Fluid Mech. 22 (2020), no. 3, Paper No. 43, 14.

[32] A. Novotný and M. Pokorný, Stabilization to equilibria of compressible Navier-Stokes equations with infinite mass, Comput. Math. Appl. 53 (2007), no. 3-4, 437-451.

[33] M. Oliver, Classical solutions for a generalized Euler equation in two dimensions, J. Math. Anal. Appl. 215 (1997), no. 2, 471-484.

[34] S. Schochet, The compressible Euler equations in a bounded domain: existence of solutions and the incompressible limit, Comm. Math. Phys. 104 (1986), no. 1, 49-75.

\author{
Nilasis Chaudhuri \\ Department of Mathematics \\ Imperial College London \\ 180 Queen's Gate, Huxley Building \\ London SW7 $2 A Z$ \\ $U K$ \\ E-mail: n.chaudhuri@imperial.ac.uk
}

Accepted: 3 January 2022 